\title{
YOUNG-MEASURE QUASI-STATIC DAMAGE EVOLUTION
}

\author{
A. FIASCHI, D. KNEES, AND U. STEFANELLI
}

\begin{abstract}
An existence result for the quasi-static evolution of incomplete damage in elastic materials is presented. The absence of gradient terms in the damage variable causes a critical lack of compactness. Therefore, the analysis is developed in the framework of Young measures, where a notion of solution is defined, presenting some improvements with respect to previous contributions. The main new feature in the proof of the existence result regards a delicate construction of the joint-recovery sequence.
\end{abstract}

\section{INTRODUCTION}

Damage processes are recurrent in Solid Mechanics. By undergoing loading cycles, real materials experience to a variable extent a deterioration of the respective elastic properties. This can be generally interpreted as the effect of the occurrence and growth of cracks and voids at the level of the microscopic material structure and has a dramatic impact on materials and structures performance. As such, damage modeling has been a remarkably active trend in the Engineering community since the 50s, so that it is largely beyond our scope even to try to review the huge existing literature on this subject. The Reader is however referred to [5, 15, 21, 22, 23] for some recent contributions.

The usual approach to damage in Continuum Mechanics is that of directly incorporating an internal variable descriptor of the state of the material into the constitutive relations. In particular, in case of isotropic damage (i.e., by assuming deterioration to be distributed with a uniform orientation), one is lead to introduce a scalar damage variable $z$ taking the value $z=1$ at undamaged points and $z=0$ at maximally damaged points. Hence, moving within the small-strain realm, one is generally concerned with an elastic energy functional of the form

$$
\mathcal{W}(z, e(v)):=\int_{\Omega} W(z(x), e(v)(x)) \mathrm{d} x
$$

where $e(v):=\left(\nabla v+\nabla v^{T}\right) / 2$ is the symmetrized strain tensor and $v: \Omega \rightarrow \mathbb{R}^{d}$ denotes the displacement from the reference configuration $\Omega$. Damage evolution is governed by the interplay of energy minimization and dissipation. In particular, damage is often very well assimilable to a quasi-static evolution process and, in this regard, the first possible choice for a dissipation mechanism from the damage state $z_{\text {old }}$ to updated state $z_{\text {new }}$ may be assumed to be

$$
\mathcal{D}\left(z_{\text {old }}, z_{\text {new }}\right):=\int_{\Omega} d\left(z_{\text {old }}(x), z_{\text {new }}(x)\right) \mathrm{d} x,
$$


where $d: \mathbb{R}^{2} \rightarrow[0, \infty]$ is the non-symmetric (pseudo-)distance defined by

$$
d\left(\theta_{1}, \theta_{2}\right):= \begin{cases}\rho\left(\theta_{1}-\theta_{2}\right) & \text { if } \theta_{1} \geq \theta_{2} \\ \infty & \text { else }\end{cases}
$$

for some $\rho>0$. The asymmetry of the dissipation distance $d$ encodes the quite natural ansatz of irreversibility of damage. Moreover, the 1-homogeneity of $\mathcal{D}$ is the trademark of the rateindependent nature of the damage process.

This very frame for a variational theory of rate-independent damage has attracted a good deal of attention among in recent years and rigorous mathematical results are to be found, for instance, in [2, 3, 14, 16, 28, 30]. The analysis of this paper moves exactly within the setting of the result by Thomas \& Mielke [35] where the Authors develop an existence theory for incomplete damage by directly including a gradient term of the internal variable $z$ into the energy. By including such gradient term, one obtains a clear compactifing effect along with the possible description of nonlocal interactions of damage in the material. On the other hand, the occurrence of damage localization seems to be often a clear experimental evidence. In this respect, one is motivated in considering possibly non-regularized damage models instead.

The novelty of our contribution with respect to [35] resides exactly in dropping the gradient term in the damage variable from the energy, thus excluding nonlocal damage interaction. Correspondingly, we are lacking the above mentioned compact frame and we resort in considering Young measures as plausible objects for describing damage evolution. Young measures are indeed a quite naturally suited tool for the treatment of non-compact problems. In particular, for rate-independent models, analyses of mechanical phenomena within the framework of Young measures have been devised in [12, 20, 24, 26, 27, 29] for phase transitions, [6, 8] for plasticity with softening, and [4] for fracture mechanics. To our knowledge, no Young measure formulation has been yet proposed in the context of rate-independent damage (in the case of a gradient-flow damage model, a Young-measure analysis at the time-discrete level is reported in [33]).

The focus of this paper is on providing an existence theory for a suitable Young-measure quasistatic evolution of the damage model in the frame of so-called energetic solutions à la MIELKE \& Theil [31]. Our evolution will be represented by a family $\nu=\left(\nu_{t}\right)_{t}$ of time-parametrized Young measures which replace the pair $(z, e(v))$. According to the expected unidirectionality of the damage process, the energetic solution is required to satisfy a suitable irreversibility property. To formulate this monotonicity condition in our generalized setting, we tailor a partial order relation between Young measures (see Section 3.1), in the same spirit as in [4]. Then, the validity of a specific global stability condition and of the energy balance will be achieved by passing to the limit argument with respect to time-discretizations.

As already commented in [35, the discontinuity of the dissipation distance makes the proof of the stability condition more complicated by requiring the construction of a so-called $m u$ tual recovery sequence. This is exactly the point where the compactifying effect of the gradient of damage in [35] has proved to be useful in order to ensure a stronger convergence of the recovery sequence. Here, we overcome this point by two tools: a regularity result and a measure-reconstruction lemma. At first, we exploit the fact that some higher integrability of the approximating sequences can be achieved by exploiting the theory of quasi-minima [17. We believe this observation (already done in [12]) to be an interesting feature of our proof which 
could possibly be of some use also elsewhere. Then, we provide a constructive technique to build a recovery sequence satisfying both the order constraint and the required convergence property.

The technical difficulties related to the Young measure approach force us to consider some reduced global stability condition. In particular, as it is quite usual in these situations, we obtain global stability for two class of competitors: translations of $\nu_{t}$ by functions $(\tilde{z}, \tilde{u})$ in $L^{1}(\Omega ; \mathbb{R}) \times H_{0}^{1}\left(\Omega ; \mathbb{R}^{d}\right)$, and Young measures with disintegration of the form $\tilde{\mu}^{x} \otimes \delta_{e}(\tilde{v})(x)$, for any Young measure $\tilde{\mu}$ on $\Omega \times[0,1]$. Minimality with respect to translations by functions coincides with the stability condition considered in [8] and [11. Here, nevertheless we allow milder assumptions on the energy density. On the other hand, the second class of tests represents a quite remarkable enlargement of the set of competitors with respect to previous contributions. These competitors, in particular, do not depend on the evolution $\nu_{t}$ and permit the comparison of the evolution with all other possible damage states.

A further interesting feature of our result is that the specific form of the damage model allows us to prove the existence result without the help of the technical tool of compatible systems of Young measures developed in [7] (see also [11]). In particular, this entails a rather straightforward formulation of our solution notion.

Our damage model is non-brittle in the sense that partially damaged situations $z \in(0,1)$ are actually to be expected (see Subsection 2.1). We shall refer to FrancForT \& Garroni [14, Garroni \& LARSEn [16], and BABADJian [2] for recent contributions on damage models for brittle materials, namely assuming $z \in\{0,1\}$. Besides brittleness, we have to remark that the mechanical stand of the latter papers is quite different from ours. In particular, their starting point is a $z$-mixture of a linearly elastic strong and weak material with elasticity tensors $A_{s}$ and $A_{w}$, respectively. This is to say that their energy density is assumed to be of the form

$$
W(z, e)= \begin{cases}A_{s} \phi(e) & \text { if } z=1 \\ A_{w} \phi(e) & \text { if } z=0 \\ +\infty & \text { otherwise }\end{cases}
$$

with $\phi(e)=e^{2} / 2$ (in the 1-dimensional case) in [14, 16] and a more general convex function $\phi$ in 2. As no gradient terms in the damage variable are considered, evolution via time-discretization immediately calls for quasi-convexification and the passage to the limit is performed by determining the limiting materials via its elasticity tensor by homogenization tools. To this end, the convexity of the energy density with respect to the strain variable is needed [2, Section 1] and a price to pay is the replacement of the damage variable $z$ by the elasticity tensor or by the damage set in the limit.

Our approach here is somehow different as we already start from an (essentially) quasiconvex energy in the first place so that no quasi-convexification is needed for the incremental step. From the one hand, this prevents us from considering linear mixtures energies of the form of (1.1) in our frame. In particular, the relaxed models from [14, 16, 2] seem not directly recoverable in the present setting. From the other hand, this gives us the advantage of tracing the damage variable $z$ into the evolution.

The paper is organized as follows. In Section 2 we present the mechanical model, and in Section 3 we recall some mathematical preliminaries. In particular, Subsection 3.1 presents a 
partial order relation between Young measures. Section 4 is devoted to the formulation of the quasi-static evolution and our main result. The existence proof is detailed in Section 5. Some technical lemmas are then collected in the Appendix.

\section{THE MECHANICAL MODEL}

Let us specify here some notation and our general assumptions. The reference configuration of the body is the bounded, connected, and open set $\Omega \subset \mathbb{R}^{d}$ with Lipschitz boundary $\partial \Omega$. We indicate the displacement field by $v$ and the linearized strain tensor by $e(v):=\frac{1}{2}\left(\nabla v+\nabla v^{\mathrm{T}}\right)$. The damage variable is $z: \Omega \rightarrow \mathbb{R}$ and will actually take values solely in $[0,1]$ as an effect of our general assumptions below.

The stored energy density of the material is a function $W: \mathbb{R} \times \mathbb{R}_{\mathrm{sym}}^{d \times d} \rightarrow[0,+\infty)$ satisfying the following hypotheses:

(W.1) $W$ is continuous and S-cross-quasiconvex, i.e. satisfies property (3.4) below;

( $W .2$ ) there exist two positive constants $c_{W}<C_{W}$ such that $c_{W}|\varepsilon|^{2} \leq W(\theta, \varepsilon) \leq C_{W}|\varepsilon|^{2}$ for every $\varepsilon \in \mathbb{R}_{\text {sym }}^{d \times d}$ and every $\theta \in(-\infty, 2]$;

$(W .3)$ for every $\theta \in \mathbb{R}, W(\theta, \cdot)$ is $C^{1}$ and $\left|\frac{\partial W}{\partial \varepsilon}(\theta, \varepsilon)\right| \leq C_{W}(|\varepsilon|+1)$, for every $(\theta, \varepsilon) \in(-\infty, 2] \times$ $\mathbb{R}_{\mathrm{sym}}^{d \times d}$

(W.4) $\theta \mapsto W(\theta, \varepsilon)$ is non-decreasing for every $\varepsilon \in \mathbb{R}_{\mathrm{sym}}^{d \times d}$;

$(W .5) W(\theta, \varepsilon)=W(0, \varepsilon)$ for every $\theta \leq 0$.

Hence, stored energy of the material reads

$$
\mathcal{W}(z, e(v)):=\int_{\Omega} W(z(x), e(v)(x)) \mathrm{d} x .
$$

Though the most natural assumption for the stored energy density in linearized elasticity is to be quadratic with respect to the strain variable, for sake of generality we assume here that $W$ satisfies the weaker condition (W.1). Indeed, our analysis could be retraced in the case of nonlinear elasticity as well, and in this case the quasi-convexity assumption is more desirable than the quadratic one.

The dissipation distance between two damage states $z_{\text {old }}$ and $z_{\text {new }}$ is given by

$$
\mathcal{D}\left(z_{\text {old }}, z_{\text {new }}\right):=\int_{\Omega} d\left(z_{\text {old }}(x), z_{\text {new }}(x)\right) \mathrm{d} x,
$$

where the density $d$ is given by

$$
d\left(\theta_{1}, \theta_{2}\right):= \begin{cases}\rho\left|\theta_{1}-\theta_{2}\right| & \text { if } \theta_{1} \geq \theta_{2} \\ +\infty & \text { else }\end{cases}
$$

for every $\theta_{1}, \theta_{2} \in \mathbb{R}$ and for a suitable $\rho>0$. 
Given two distinct times $s<t$, the global dissipation of a possibly discontinuous-in-time damage evolution $z:[0, T] \rightarrow L^{1}(\Omega)$ in the interval $[s, t]$ is given by

$$
\operatorname{Diss}(z ; s, t):=\sup \sum_{i=1}^{k} \mathcal{D}\left(z\left(\tau_{i-1}\right),\left(z\left(\tau_{i}\right)\right),\right.
$$

where the supremum is taken among all finite partitions $s=\tau_{0}<\tau_{1}<\cdots<\tau_{k}=t$.

Note that, if $z(\tau) \geq z\left(\tau^{\prime}\right)$ almost everywhere in $\Omega$, whenever $\tau \leq \tau^{\prime}$, then

$$
\operatorname{Diss}(z ; s, t)=\rho \int_{\Omega}(z(s)-z(t)) \mathrm{d} x
$$

For the sake of simplicity, the boundary displacement is prescribed at time $t$ on the whole boundary $\partial \Omega$ as $u=\varphi$ where the given function $\varphi(t)$ fulfills

$$
\varphi \in A C\left([0, T] ; W^{1, p}\left(\Omega ; \mathbb{R}^{d}\right)\right), \quad \text { with } 2<p \leq \infty .
$$

Let us however note that other choices of boundary conditions are indeed possible.

2.1. A 0-dimensional example. We focus here on a 0 -dimensional case, i.e. the case in which damage and strain are independent of $x$. Our aim is showing that the materials we are considering are not necessarily brittle, in the sense that the damage variable $z$ can be expected to take intermediate values between 0 and 1 .

We consider a stored energy defined by

$$
W(z, e):=\frac{e^{2}}{2 g(z)},
$$

for $g(z):=\sqrt{2-z^{+}}$for every $z \in[0,2)$. We observe that the function $g$ is $C^{2}(0,2)$ with $g^{\prime} \leq 0$ and $g^{\prime \prime} \leq 0$ in $(0,2)$ and $g$ is constant on $(-\infty, 0]$; it is now easy to see that the Hessian matrix of $W$ is positive definite and hence $W$ is a convex function on $[0,2) \times \mathbb{R}$ (see [35, Lemma 5.1]).

The dissipation distance is given by

$$
d\left(z_{1}, z_{2}\right):= \begin{cases}\left|z_{1}-z_{2}\right| & \text { if } z_{1} \geq z_{2} \\ +\infty & \text { otherwise }\end{cases}
$$

for every $z_{1}, z_{2} \in \mathbb{R}$.

In this example we analyze an evolution driven by time-dependent external forces instead of time-varying boundary data; the external forces are given by $l(t):=t$.

In particular, a quasi-static evolution in the time interval $[0,3 \sqrt{2}]$ with initial datum $\left(z_{0}, e_{0}\right):=$ $(1,0)$ is defined energetically (see [31]) as a pair of time-dependent functions $(z(t), e(t))$ with 
$z(t) \geq 0$, such that the following conditions are satisfied:

$$
\begin{aligned}
& \text { initial condition : }(z(0), e(0))=(1,0) \\
& \text { irreversibility }: z(s) \geq z(t) \text { if } s \leq t \\
& \text { stability : } \frac{e^{2}(t)}{2 g(z(t))}-t e(t) \leq \frac{\tilde{e}^{2}}{2 g(\tilde{z})}-t \tilde{e}+d(z(t), \tilde{z}) \forall t \in[0,3 / \sqrt{2}], \tilde{z}, \tilde{e} \in \mathbb{R} \text {; } \\
& \text { energy equality : } \frac{e^{2}(t)}{2 g(z(t))}-t e(t)+z(0)-z(t)=-\int_{0}^{t} e(s) \mathrm{d} s \quad \forall t \in[0,3 / \sqrt{2}] .
\end{aligned}
$$

Condition (2.3) implies that $e(t)=t g(z(t))$. Indeed, if we choose $\tilde{z}=z(t)$ in (2.3), we obtain that $e(t)$ is the unique minimizer of the convex function $e \mapsto e^{2} /(2 g(z(t)))-t e$. Therefore, it is enough to choose $z(t)$ satisfying the initial condition and the irreversibility condition, such that the energy equality 2.4 holds true for $(z(t), \operatorname{tg}(z(t)))$, and satisfying for every $t \in[0,3 \sqrt{2}]$

$$
\frac{t^{2} g(z(t))}{2}-t^{2} g(z(t)) \leq \frac{t^{2} g(\tilde{z})}{2}-t^{2} g(\tilde{z})+z(t)-\tilde{z},
$$

for every $\tilde{z} \leq z(t)$, i.e.,

$$
\frac{t^{2}}{2}[g(\tilde{z})-g(z(t))] \leq z(t)-\tilde{z}
$$

for every $\tilde{z} \leq z(t)$.

Let us first consider $z(t) \equiv 1$ for every $t \in[0,2]$. This choice may be easily proved to fulfill (2.1)-(2.4) and hence is a quasi-static evolution for $t \in[0,2]$. We want to show that, for $t>2$, $z(t)=1$ does not satisfy the stability condition 2.5) and hence $z(t)$ has to be strictly smaller than 1 . We rephrase this by saying that there exists $\tilde{z} \in[0,1]$ such that $f(\tilde{z})>0$ where $f$ is given by

$$
f(\tilde{z}):=\frac{t^{2}}{2}[g(\tilde{z})-1]-1+\tilde{z} .
$$

Indeed, let us consider $\tilde{z}_{t}:=t^{2}-t^{4} / 4+1=\left(8-\left(t^{2}-2\right)^{2}\right) / 4$. We observe that $\tilde{z}_{t} \in(0,1)$ and $f\left(\tilde{z}_{t}\right)=0$ if $t \in(2,3 / \sqrt{2})$. Moreover,

$$
f^{\prime}\left(\tilde{z}_{t}\right)=-\frac{t^{2}}{4 \sqrt{2-\tilde{z}_{t}}}+1=-\frac{t^{2}}{4 \sqrt{2-\left(t^{2}-\frac{t^{4}}{4}+1\right)}}+1=-\frac{t^{2}}{4\left(\frac{t^{2}}{2}-1\right)}+1=\frac{t^{2}-4}{2\left(t^{2}-2\right)}>0
$$

since $t>2$. Therefore, there exists $\tilde{z} \in\left(\tilde{z}_{t}, 1\right)$ such that $f(\tilde{z})>0$. Hence, $z(t)=1$ does not fulfill the stability condition 2.5 for $t \in(2,3 / \sqrt{2})$ and we will necessarily have $z(t) \in(-\infty, 1)$. 
On the other hand, we cannot have $z(t)=1$ for $t \in[0,2]$ and $z(t) \leq 0$ for $t \in(2,3 / \sqrt{2})$, because in this case the energy balance for $s \in(2,3 / \sqrt{2})$ would not be fulfilled as

$$
\begin{aligned}
& \frac{s^{2} g(0)}{2}-s^{2} g(0)+1-z(t)+\int_{0}^{s} t g(z(t)) \mathrm{d} t=-\frac{s^{2} \sqrt{2}}{2}+1-z(t)+\int_{0}^{2} t \mathrm{~d} t+\int_{2}^{s} t \sqrt{2} \mathrm{~d} t \\
& =-\frac{s^{2} \sqrt{2}}{2}+1-z(t)+2+\frac{s^{2} \sqrt{2}}{2}-2 \sqrt{2}=1-z(t)-2(\sqrt{2}-1)>0 .
\end{aligned}
$$

Eventually, we have proved that there exists $t \in(2,3 / \sqrt{2})$ with $z(t) \in(0,1)$.

\section{Mathematical PRELiminaries}

Let $\mathcal{L}^{d}$ denote the Lebesgue measure on $\mathbb{R}^{d}, d \geq 1$. We sometimes use the notation $|E|$ for the Lebesgue measure of the measurable subset $E \subseteq \mathbb{R}^{d}$ as well. Throughout the paper $\Omega$ will be a bounded, connected, open subset of $\mathbb{R}^{d}$ with Lipschitz boundary. The Borel $\sigma$-algebra on $\Omega$ is denoted by $\mathcal{B}(\Omega)$. For $1 \leq p \leq \infty,\|\cdot\|_{p}$ stands for the usual norm on $L^{p}, W^{1, p}\left(\Omega ; \mathbb{R}^{d}\right)$ denotes the usual Sobolev space, $H^{1}\left(\Omega ; \mathbb{R}^{d}\right):=W^{1,2}\left(\Omega ; \mathbb{R}^{d}\right)$, and the symbol $\langle\cdot, \cdot\rangle$ is the scalar product in $H^{1}$, if not otherwise specified. Given a function $f \in L^{1}(\Omega)$ and a measurable $Q \subseteq \Omega$, the mean value of $f$ over $Q$ is denoted by $(f)_{Q}$, i.e.,

$$
(f)_{Q}:=\frac{1}{|Q|} \int_{Q} f(x) \mathrm{d} x .
$$

We indicate the positive part of a function $f$ with $f^{+}:=f \vee 0$.

We recall the notion of quasi-minima of integral functionals. Given $\varphi \in H^{1}\left(\Omega ; \mathbb{R}^{d}\right)$, let $\mathcal{G}$ be the functional defined by

$$
\mathcal{G}(v)=\mathcal{G}(v, \Omega):=\int_{\Omega} G(x, \nabla v(x)) \mathrm{d} x
$$

for every $v \in \varphi+H_{0}^{1}\left(\Omega ; \mathbb{R}^{d}\right)$, where $G: \Omega \times \mathbb{R}^{d \times d} \rightarrow \mathbb{R}$ is a Carathéodory function satisfying

$$
\begin{aligned}
& G(x, F) \leq L\left(|F|^{2}+1\right) \\
& G(x, F) \geq \tilde{G}(F)-l
\end{aligned}
$$

for suitable positive constants $L, l$, for every $(x, F) \in \Omega \times \mathbb{R}^{d \times d}$, where $\tilde{G}: \mathbb{R}^{d \times d} \rightarrow \mathbb{R}$ satisfies the following estimate:

$$
\exists K>0: \quad \int_{\Omega} \tilde{G}(\nabla \phi(x)) \mathrm{d} x \geq K\|\nabla \phi\|_{2}^{2} \quad \text { for every } \phi \in H_{0}^{1}\left(\Omega ; \mathbb{R}^{d}\right)
$$

Definition 3.1. (Quasi-minimum, [17]) Let $V \in W^{1, p}\left(\Omega ; \mathbb{R}^{d}\right)$ and $\lambda>0$. A function $v \in$ $V+H_{0}^{1}\left(\Omega ; \mathbb{R}^{d}\right)$ is said to be a cubic $\lambda$-quasi-minimum for the functional $\mathcal{G}$ if for every cube of side $R, Q_{R} \subset \mathbb{R}^{d}$, and every $w \in H^{1}\left(\Omega ; \mathbb{R}^{d}\right)$ such that $v-w \in H_{0}^{1}\left(\Omega \cap Q_{R}\right)$ we have

$$
\int_{\left(Q_{R} \cap \Omega\right)} G(x, \nabla v(x)) \mathrm{d} x \leq \lambda \int_{Q_{R} \cap \Omega} G(x, \nabla v(x)) \mathrm{d} x .
$$


Theorem 3.2. (Higher integrability, [18, Chapter 6]) Let $V \in W^{1, p}\left(\Omega ; \mathbb{R}^{d}\right)$, for $2<p$, and let $v \in V+H_{0}^{1}\left(\Omega ; \mathbb{R}^{d}\right)$ be a $\lambda$-cubic quasi-minimum of the functional $\mathcal{G}$. Then, there exist constants $\gamma>0$ and $r>1$, depending only on $\lambda$ and $V$, such that

$$
\int_{\Omega}|\nabla v|^{2 r} \mathrm{~d} x \leq \gamma\left\{\left(\int_{\Omega}|\nabla v|^{2} \mathrm{~d} x\right)^{r}+1\right\} .
$$

We recall the statement of the Korn-Poincaré inequality (see [34]): for every open, Lipschitz set $D \subset \mathbb{R}^{d}$, there exists a positive constant $C(D)$ such that

$$
\|\nabla v\|_{H^{1}(D)} \leq C(D)\|e(v)\|_{L^{2}(D)},
$$

for every $v \in H_{0}^{1}(D)$.

We recall the definition of cross-quasiconvexity in the form used in [13] and a related semicontinuity result ([13, Theorem 4.4]). A continuous function $G: \mathbb{R} \times \mathbb{R}^{d \times d} \rightarrow \mathbb{R}$ is cross-quasiconvex if for every $\theta \in[0,1], F \in \mathbb{R}^{d \times d}$ we have

$$
G(\theta, F) \leq \frac{1}{|\Omega|} \int_{\Omega} G(\theta+m(x), F+\nabla u(x)),
$$

for every $u \in H_{0}^{1}\left(\Omega ; \mathbb{R}^{d}\right)$ and every $m \in L^{\infty}(\Omega)$, with $\theta+m(x) \in[0,1]$ for a.e. $x \in \Omega$ and $\int_{\Omega} m(x) \mathrm{d} x=0$.

Lemma 3.3. (Lower semicontinuity) If $G: \mathbb{R} \times \mathbb{R}^{d \times d} \rightarrow \mathbb{R}$ is cross-quasi-convex and fulfills

$$
0 \leq G(\theta, F) \leq g(\theta)\left(1+|F|^{2}\right)
$$

for every $\theta \in \mathbb{R}, F \in \mathbb{R}^{d \times d}$, and some $g \in L_{\text {loc }}^{\infty}(\mathbb{R})$ we have that

$$
\int_{\Omega} G(z(x), \nabla v(x)) \mathrm{d} x \leq \liminf _{k} \int_{\Omega} G\left(z_{k}(x), \nabla v_{k}(x)\right) \mathrm{d} x,
$$

whenever $z_{k} \rightarrow z L^{\infty}$-weakly*, $z_{k}(x) \in[0,1]$ for a.e. $x \in \Omega$, and $v_{k} \rightarrow v$ weakly in $H^{1}\left(\Omega ; \mathbb{R}^{d}\right)$.

Note that if $H: \mathbb{R} \times \mathbb{R}_{\mathrm{sym}}^{d \times d} \rightarrow \mathbb{R}$ is a continuous function satisfying

$$
\begin{gathered}
0 \leq H(\theta, \varepsilon) \leq g(\theta)\left(1+|\varepsilon|^{2}\right) \quad \text { for } g \in L_{\mathrm{loc}}^{\infty}(\mathbb{R}) ; \\
H(\theta, \varepsilon) \leq \frac{1}{|\Omega|} \int_{\Omega} H(\theta+m(x), \varepsilon+e(u)(x)),
\end{gathered}
$$

for every $u \in H_{0}^{1}\left(\Omega ; \mathbb{R}^{d}\right), m \in L^{\infty}(\Omega ; \mathbb{R})$ with $\int_{\Omega} m(x) \mathrm{d} x=0$ and $\theta+m(x) \in[0,1]$ for a.e. $x \in \Omega$, then the function $G(\theta, F):=H(\theta, S(F))$, with $S(\theta, F):=\left(\theta, \frac{F+F^{T}}{2}\right)$, satisfies properties $(3.2)$ and (3.3). We will say that a function satisfying (3.4) is $S$-cross-quasiconvex.

We define $M_{b}\left(\Omega \times \mathbb{R}^{N}\right)$ as the space of bounded Radon measures on $\Omega \times \mathbb{R}^{N}$. This space can be identified with the dual of the Banach space $C_{0}\left(\Omega \times \mathbb{R}^{N}\right)$ of all continuous functions $\phi: \Omega \times \mathbb{R}^{N} \rightarrow \mathbb{R}$ such that $|\phi| \geq \varepsilon$ is compact for every $\varepsilon>0$. We will consider on $M_{b}\left(\Omega \times \mathbb{R}^{N}\right)$ the weak* topology deriving from this duality.

Let us refer to [36] for a general introduction on Young measures and just recall some definition and fix notation. A Young measure $\mu \in Y\left(\Omega ; \mathbb{R}^{N}\right)$ is a nonnegative measure in $M_{b}\left(\Omega \times \mathbb{R}^{N}\right)$, 
such that $\pi_{\Omega}(\mu)=\mathcal{L}^{d}$, where $\pi_{\Omega}(x, \xi):=x$. By the Disintegration Theorem, one can associate to $\mu$ a measurable family of probability measures $\left(\mu^{x}\right)_{x \in \Omega}$ on $\mathbb{R}^{N}$ in such a way that

$$
\int_{\Omega \times \mathbb{R}^{N}} f(x, \xi) d \mu(x, \xi)=\int_{\Omega}\left(\int_{\mathbb{R}^{N}} f(x, \xi) \mathrm{d} \mu^{x}(\xi)\right) \mathrm{d} x
$$

for every bounded Borel function $f: \Omega \times \mathbb{R}^{N} \rightarrow \mathbb{R}$. We define the barycentre of $\mu$ as the function

$$
\operatorname{bar}(\mu)(x):=\int_{\mathbb{R}^{N}} \xi \mathrm{d} \mu^{x}(\xi) \text { for a.e. } x \in \Omega,
$$

and the $p$-moment of $\mu$, for $1<p \leq \infty$, as the quantity

$$
\int_{\Omega \times \mathbb{R}^{N}}|\xi|^{p} \mathrm{~d} \mu(x, \xi) .
$$

We denote by $Y^{p}\left(\Omega ; \mathbb{R}^{N}\right)$ the set of measures in $Y\left(\Omega ; \mathbb{R}^{N}\right)$ with finite $p$-moments. Given a sequence $\left(\mu_{k}\right)_{k}$ in $Y\left(\Omega ; \mathbb{R}^{N}\right)$, we say that $\mu_{k} \rightarrow \mu p$-weakly*, for $1<p \leq \infty$, if

$$
\begin{gathered}
\mu_{k} \rightarrow \mu \text { in the weak* topology of } M_{b}\left(\Omega \times \mathbb{R}^{N}\right), \\
\int_{\Omega \times \mathbb{R}^{n}}|\xi|^{p} d \mu_{k}(x, \xi) \text { are equibounded in } k .
\end{gathered}
$$

Let $(D, \mathcal{F})$ be a measure space and $\mu \in Y\left(\Omega ; \mathbb{R}^{N}\right)$. For every $\mathcal{B}\left(\Omega \times \mathbb{R}^{N}\right)-\mathcal{F}$-measurable function $f: \Omega \times \mathbb{R}^{N} \rightarrow D$, the image measure, defined by $\mu\left(f^{-1}(B)\right)$ for every measurable set $B \subseteq D$, will be denoted by $f(\mu)$. In particular, if we define the translation map $\operatorname{Tr}_{G}$ associated to a function $G \in L^{1}\left(\Omega ; \mathbb{R}^{N}\right)$ by

$$
\operatorname{Tr}_{G}(x, \xi):=(x, \xi+G(x)), \quad \text { for a.e. } x \in \Omega \text { and every } \xi \in \mathbb{R}^{N},
$$

for every measure $\mu \in Y\left(\Omega ; \mathbb{R}^{N}\right)$ we can consider the translated measure $\operatorname{Tr}_{G}(\mu)$, defined by

$$
\int_{\Omega \times \mathbb{R}^{N}} \phi(x, \xi) d \operatorname{Tr}_{G}(\mu)(x, \xi)=\int_{\Omega \times \mathbb{R}^{N}} \phi(x, \xi+G(x)) d \mu(x, \xi),
$$

for every bounded Borel function $\phi: \Omega \times \mathbb{R}^{N} \rightarrow \mathbb{R}$.

Given $\xi_{0} \in \mathbb{R}^{N}$, the measure $\delta_{\xi_{0}} \in M_{b}\left(\mathbb{R}^{N}\right)$ is classically defined by

$$
\int_{\mathbb{R}^{N}} f(\xi) d \delta_{\xi_{0}}(\xi)=f\left(\xi_{0}\right)
$$

for every bounded Borel function $f: \mathbb{R}^{N} \rightarrow \mathbb{R}$. Fixed a $\mathcal{B}(\Omega)-\mathcal{B}\left(\mathbb{R}^{N}\right)$-measurable function $u: \Omega \rightarrow \mathbb{R}^{N}$, the Young measure $\boldsymbol{\delta}_{u} \in Y\left(\Omega ; \mathbb{R}^{N}\right)$ is defined by

$$
\int_{\Omega \times \mathbb{R}^{N}} g(x, \xi) d \boldsymbol{\delta}_{u}(x, \xi)=\int_{\Omega} g(x, u(x)) d x,
$$

for every bounded Borel function $g: \Omega \times \mathbb{R}^{N} \rightarrow \mathbb{R}$.

The following lemma is a slight modification of [32, Proposition 6.5, pg. 103]. 
Lemma 3.4. (Continuity) Let $1<p \leq \infty$, and let $\left(\mu_{k}\right)_{k} \subseteq Y^{p}\left(\Omega ; \mathbb{R}^{N}\right)$ converge $p$-weakly* to $\mu \in Y^{p}\left(\Omega ; \mathbb{R}^{N}\right)$. Then, for every Carathéodory function $f: \Omega \times \mathbb{R}^{N} \rightarrow \mathbb{R}$, with $|f(x, \xi)| \leq$ $a(x)+b(x)|\xi|^{q}$, for every $x \in \Omega, \xi \in \mathbb{R}^{N}, 1 \leq q<p, b \in L^{p /(p-q)}(\Omega)$, and $a \in L^{1}(\Omega)$, it holds

$$
\int_{\Omega \times \mathbb{R}^{N}} f(x, \xi) d \mu_{k}(x, \xi) \longrightarrow \int_{\Omega \times \mathbb{R}^{N}} f(x, \xi) d \mu(x, \xi) .
$$

Finally, we recall that a measure $\nu \in Y^{p}\left(\Omega ; \mathbb{R}^{d \times d}\right)$ is a $W^{1, p}$-gradient Young measure (see, e.g., [19]) for $p>1$ if there exists a bounded sequence $\left(v_{n}\right)_{n} \in W^{1, p}\left(\Omega ; \mathbb{R}^{d}\right)$ such that $\boldsymbol{\delta}_{\nabla v_{n}} \rightarrow \nu$ $p$-weakly* as $n \rightarrow \infty$. For the characterization and the properties of such measures we refer to [32].

Note that, thanks to Lemma 3.4 , given a bounded sequence $\left(v_{n}\right)_{n}$ in $W^{1, p}\left(\Omega ; \mathbb{R}^{d}\right)$ with $\boldsymbol{\delta}_{\nabla v_{n}} \rightarrow \nu p$-weakly*, for $p>1$, we have that $\boldsymbol{\delta}_{e\left(v_{n}\right)} \rightarrow S \nu p$-weakly*, where we recall that $S: \Omega \times \mathbb{R}^{d \times d} \rightarrow \Omega \times \mathbb{R}_{\mathrm{sym}}^{d \times d}$ is defined by $S(x, F):=\left(x, \frac{F+F^{T}}{2}\right)$, for every $x \in \Omega$ and $F \in \mathbb{R}^{d \times d}$.

Henceforth $C$ will stand for any positive constant, possibly depending on data and varying from line to line.

3.1. An order relation between Young measures. In this section we want to define an order relation on the set $Y(\Omega ;[0,1])$ of the Young measures on $\Omega$ with values in $\mathbb{R}$ and support contained in $\Omega \times[0,1]$.

Definition 3.5. (Order) Given $\mu_{1}, \mu_{2} \in Y(\Omega ;[0,1])$, we write $\mu_{1} \succeq \mu_{2}$ if

$$
\mu_{1}^{x}(\alpha, \infty) \geq \mu_{2}^{x}(\alpha, \infty) \text { for a.e. } x \in \Omega \text { and for every } \alpha \in \mathbb{R} \text {. }
$$

It is easy to see that $\succeq$ is an order and that, in the case of $\mu_{1}=\boldsymbol{\delta}_{z^{1}}$ and $\mu_{2}=\boldsymbol{\delta}_{z_{2}}$ for some measurable functions $z^{1}, z^{2}: \Omega \rightarrow[0,1]$, we have $\boldsymbol{\delta}_{z^{1}} \succeq \boldsymbol{\delta}_{z^{2}}$ if and only if $z^{1} \geq z^{2}$ almost everywhere in $\Omega$.

Now we give an equivalent characterization of this order relation.

Theorem 3.6. (Order characterization) Given two Young measures $\mu_{1}, \mu_{2} \in Y(\Omega ;[0,1])$, we have $\mu_{1} \succeq \mu_{2}$ if and only if there exists $\mu_{12} \in Y\left(\Omega ;[0,1]^{2}\right)$ such that

$$
\begin{gathered}
\pi_{1}\left(\mu_{12}\right)=\mu_{1}, \quad \pi_{2}\left(\mu_{12}\right)=\mu_{2}, \\
\mu_{12}^{x}\left(\left\{\theta_{1}<\theta_{2}\right\}\right)=0 \text { for a.e. } x \in \Omega,
\end{gathered}
$$

where $\pi_{1}\left(x, \theta_{1}, \theta_{2}\right):=\left(x, \theta_{1}\right), \pi_{2}\left(x, \theta_{1}, \theta_{2}\right):=\left(x, \theta_{2}\right)$, for every $\left(x, \theta_{1}, \theta_{2}\right) \in \Omega \times \mathbb{R}^{2}$.

Proof. Let us first prove the necessity. If $\mu_{1} \nsucceq \mu_{2}$, then there exists a measurable set $E \subseteq \Omega$ with positive measure, such that $\mu_{1}^{x}\left(\left(-\infty, \alpha_{x}\right]\right)>\mu_{2}^{x}\left(\left(-\infty, \alpha_{x}\right]\right)$, for $x \in E$, for a suitable $\alpha_{x} \in[0,1]$. This implies that, for every $\mu_{12}$ satisfying the projection properties (3.6), we have $\mu_{12}^{x}\left(\left\{\theta_{1}<\theta_{2}\right\}\right)>0$ for $x \in E$. Indeed, for every $x \in E$ we have

$$
\mu_{12}^{x}\left([0,1] \times\left[0, \alpha_{x}\right]\right)=\mu_{2}^{x}\left(\left(-\infty, \alpha_{x}\right]\right)<\mu_{1}^{x}\left(\left(-\infty, \alpha_{x}\right]\right)=\mu_{12}^{x}\left(\left[0, \alpha_{x}\right] \times[0,1]\right) ;
$$

and this implies

$$
\begin{gathered}
0 \leq \mu_{12}^{x}\left(\left(\alpha_{x}, 1\right] \times\left[0, \alpha_{x}\right]\right)=\mu_{12}^{x}\left([0,1] \times\left[0, \alpha_{x}\right]\right)-\mu_{12}^{x}\left(\left[0, \alpha_{x}\right] \times\left[0, \alpha_{x}\right]\right)< \\
<\mu_{12}^{x}\left(\left[0, \alpha_{x}\right] \times[0,1]\right)-\mu_{12}^{x}\left(\left[0, \alpha_{x}\right] \times\left[0, \alpha_{x}\right]\right)=\mu_{12}^{x}\left(\left[0, \alpha_{x}\right] \times\left(\alpha_{x}, 1\right]\right) \leq \mu_{12}^{x}\left(\left\{\theta_{1}<\theta_{2}\right\}\right),
\end{gathered}
$$


for every $x \in E$.

Now we prove the sufficiency of inequality (3.5). We fix $n \in \mathbb{N}$ and consider the measures $\mu_{1, n}, \mu_{2, n}$ whose disintegration is defined by

$$
\begin{aligned}
& \mu_{1, n}^{x}:=\mu_{1}^{x}\left(\left[0, \frac{1}{n}\right]\right) \delta_{\frac{1}{n}}+\sum_{i=2}^{n} \mu_{1}^{x}\left(\left(\frac{i-1}{n}, \frac{i}{n}\right]\right) \delta_{\frac{i}{n}}, \\
& \mu_{2, n}^{x}:=\mu_{2}^{x}\left(\left[0, \frac{1}{n}\right]\right) \delta_{\frac{1}{n}}+\sum_{i=2}^{n} \mu_{2}^{x}\left(\left(\frac{i-1}{n}, \frac{i}{n}\right]\right) \delta_{\frac{i}{n}} .
\end{aligned}
$$

Since $\left(\mu_{1, n}^{x}\right)_{x}$ and $\left(\mu_{2, n}^{x}\right)_{x}$ are measurable families of probability measures on $[0,1]$, we have that $\mu_{1, n}, \mu_{2, n} \in Y(\Omega ;[0,1])$.

Moreover, $\mu_{1, n} \rightarrow \mu_{1}$ and $\mu_{2, n} \rightarrow \mu_{2}$ weakly*, as $n \rightarrow \infty$. Indeed, let $f \in C_{0}(\Omega \times \mathbb{R})$; since $f$ is uniformly continuous, there exists a modulus of continuity $\omega_{f}: \mathbb{R} \rightarrow \mathbb{R}$ such that for every $\left(x_{1}, \theta_{1}, \xi_{1}\right),\left(x_{2}, \theta_{2}, \xi_{2}\right) \in \Omega \times \mathbb{R}^{2}$

$$
\left|f\left(x_{1}, \theta_{1}, \xi_{1}\right)-f\left(x_{2}, \theta_{2}, \xi_{2}\right)\right| \leq \omega_{f}\left(\left|\left(x_{1}, \theta_{1}, \xi_{1}\right)-\left(x_{2}, \theta_{2}, \xi_{2}\right)\right|\right), \text { and } \lim _{\delta \rightarrow 0} \omega_{f}(\delta)=0
$$

Therefore, we have for $h=1,2$

$$
\begin{aligned}
& \left|\int_{\Omega \times \mathbb{R}} f(x, \theta) \mathrm{d} \mu_{h, n}(x, \theta)-\int_{\Omega \times \mathbb{R}} f(x, \theta) \mathrm{d} \mu_{h}(x, \theta)\right| \\
= & \left|\int_{\Omega}\left(\int_{\mathbb{R}} f(x, \xi) \mathrm{d} \mu_{h, n}^{x}(\theta)-\int_{\mathbb{R}} f(x, \theta) \mathrm{d} \mu_{h}^{x}(\theta)\right) \mathrm{d} x\right| \\
\leq & \int_{\Omega} \mid \mu_{h}^{x}\left(\left[0, \frac{1}{n}\right]\right) f\left(x, \frac{1}{n}\right)+\sum_{i=2}^{n} \mu_{h}^{x}\left(\left(\frac{i-1}{n}, \frac{i}{n}\right]\right) f\left(x, \frac{i}{n}\right) \\
& \left.-\int_{\mathbb{R}} f(x, \theta) \mathrm{d} \mu_{h}^{x}(\theta)\right) \mid \mathrm{d} x \\
\leq & \int_{\Omega}\left(\int_{\left[0, \frac{1}{n}\right]}\left|f\left(x, \frac{1}{n}\right)-f(x, \theta)\right| \mathrm{d} \mu_{h}^{x}(\theta)\right) \mathrm{d} x \\
& +\int_{\Omega}\left(\sum_{i=2}^{n} \int_{\left(\frac{i-1}{n}, \frac{i}{n}\right]}\left|f\left(x, \frac{i}{n}\right)-f(x, \theta)\right| \mathrm{d} \mu_{h}^{x}(\theta)\right) \mathrm{d} x \\
\leq & \omega_{f}(1 / n) \int_{\Omega} \mu_{h}^{x}([0,1]) \mathrm{d} x=\omega_{f}(1 / n)|\Omega| \rightarrow 0 \text { as } n \rightarrow \infty .
\end{aligned}
$$

For almost every $x \in \Omega$, we set

$$
\begin{array}{lll}
A_{1}^{x}:=\mu_{1, n}^{x}\left(\left[0, \frac{1}{n}\right]\right), & A_{i}^{x}:=\mu_{1, n}^{x}\left(\left(\frac{i}{n}, \frac{i+1}{n}\right]\right) & \text { for every } i=2, \ldots, n, \\
B_{1}^{x}:=\mu_{2, n}^{x}\left(\left[0, \frac{1}{n}\right]\right), & B_{j}^{x}:=\mu_{2, n}^{x}\left(\left(\frac{j}{n}, \frac{j+1}{n}\right]\right) & \text { for every } j=2, \ldots, n .
\end{array}
$$


Since $\mu_{1} \succeq \mu_{2}$, we deduce that

$$
\begin{aligned}
& \sum_{i=1}^{k} A_{i}^{x} \leq \sum_{j=1}^{k} B_{j}^{x} \quad \text { for every } k=1, \ldots, n, \\
& \sum_{i=1}^{n} A_{i}^{x}=\mu_{1}^{x}([0,1])=1=\mu_{2}^{x}([0,1])=\sum_{j=1}^{n} B_{j}^{x}, \\
& 0 \leq A_{i}^{x} \leq 1, \quad 0 \leq B_{j}^{x} \leq 1, \quad \text { for every } i \text { and } j,
\end{aligned}
$$

for almost every $x \in \Omega$. Hence $\left(A_{i}^{x}\right)_{i}$ and $\left(B_{j}^{x}\right)_{j}$ satisfy the hypotheses of Theorem 5.6 in Appendix A, and we can find a matrix $\left(C_{i j}^{x}\right)_{i j}$ with measurable entries in $[0,1]$ such that

$$
\begin{aligned}
\sum_{i=1}^{n} C_{i j}^{x} & =B_{j}^{x}, \\
\sum_{j=1}^{n} C_{i j}^{x} & =A_{i}^{x}, \\
C_{i j}^{x} & =0 \text { if } i<j .
\end{aligned}
$$

Let us define

$$
\mu_{12, n}^{x}:=\sum_{i, j=1}^{n} C_{i j}^{x} \delta_{\left(\frac{i}{n}, \frac{j}{n}\right)},
$$

for almost every $x \in \Omega$. We have, therefore, that $\mu_{12, n}^{x}\left([0,1]^{2}\right)=\sum_{i j} C_{i j}^{x}=\sum_{i} A_{i}^{x}=\sum_{j} B_{j}^{x}=1$, and $x \mapsto \mu_{12, n}^{x}(E)$ is measurable for every Borel set $E$. Hence, $\left(\mu_{12, n}^{x}\right)_{x}$ represents the disintegration of a Young measure in $Y\left(\Omega ;[0,1]^{2}\right)$. Thanks to conditions $3.8,3.9$, and $(3.10$, we have

$$
\begin{aligned}
\mu_{12, n}^{x}\left(\left\{\theta_{1}<\theta_{2}\right\}\right) & =\sum_{i<j} C_{i j}=0, \\
{\left[\pi_{1}\left(\mu_{12, n}\right)\right]^{x} } & =\sum_{i j} C_{i j}^{x} \delta_{\frac{i}{n}}=\sum_{i}\left(\sum_{j} C_{i j}^{x}\right) \delta_{\frac{i}{n}}=\sum_{i} A_{i}^{x} \delta_{\frac{i}{n}}=\mu_{1, n}^{x}, \\
{\left[\pi_{2}\left(\mu_{12, n}\right)\right]^{x} } & =\sum_{i j} C_{i j}^{x} \delta \frac{j}{n}=\sum_{j}\left(\sum_{i} C_{i j}^{x}\right) \delta_{\frac{j}{n}}=\sum_{j} B_{j}^{x} \delta_{\frac{j}{n}}=\mu_{2, n}^{x},
\end{aligned}
$$

for almost every $x \in \Omega$. Since $\left(\mu_{12, n}\right)_{n}$ are Young measures with compact support and hence have equibounded moments of every order, we can always find a subsequence $\left(\mu_{12, n_{k}}\right)_{k}$ and a Young measure $\mu_{12} \in Y\left(\Omega ;[0,1]^{2}\right)$ such that $\mu_{12, n} \rightarrow \mu_{12}$ weakly*. Since $\mu_{1, n} \rightarrow \mu_{1}$ and $\mu_{2, m} \rightarrow \mu_{2}$ weakly*, thanks to the projections properties $(3.12)$ and $(3.13)$, we deduce that

$$
\pi_{1}\left(\mu_{12}\right)=\mu_{1} \quad \pi_{2}\left(\mu_{12}\right)=\mu_{2},
$$

and hence $\mu_{12}$ satisfies the projection property (3.6). Eventually, we observe that for every open subset $E$ of $\Omega, E \times\left\{\theta_{1}<\theta_{2}\right\}$ is open, and hence $\mu_{12}\left(E \times\left\{\theta_{1}<\theta_{2}\right\}\right) \leq \liminf _{k} \mu_{12, n_{k}}\left(E \times\left\{\theta_{1}<\right.\right.$ $\left.\left.\theta_{2}\right\}\right)=0$, thanks to identity (3.11). This implies that $\mu_{12}^{x}\left(\left\{\theta_{1}<\theta_{2}\right\}\right)=0$ for almost every $x \in \Omega$, i.e. 3.7 . 
Remark 3.7. (Order of the barycentres) Note that $\mu_{1} \succeq \mu_{2}$ implies $\operatorname{bar}\left(\mu_{1}\right) \geq \operatorname{bar}\left(\mu_{2}\right)$ a.e. in $\Omega$, whereas the opposite implication is false. Indeed, if $\mu_{1} \succeq \mu_{2}$, by Lemma 3.6 there exists $\mu_{12} \in Y\left(\Omega ;[0,1]^{2}\right)$ with $\pi_{i}\left(\mu_{12}\right)=\mu_{i}, i=1,2$ and $\mu_{12}^{x}\left(\left\{\theta_{1}<\theta_{2}\right\}\right)=0$, a.e. $x \in \bar{\Omega}$; in particular we have

$$
\begin{aligned}
& \int_{E}\left[\operatorname{bar}\left(\mu_{1}\right)-\operatorname{bar}\left(\mu_{2}\right)\right] \mathrm{d} x=\int_{E \times[0,1]} \theta_{1} \mathrm{~d} \mu_{1}\left(x, \theta_{1}\right)-\int_{E \times[0,1]} \theta_{2} \mathrm{~d} \mu\left(x, \theta_{2}\right) \\
= & \int_{E \times[0,1]^{2}}\left(\theta_{1}-\theta_{2}\right) \mathrm{d} \mu_{12}\left(x, \theta_{1}, \theta_{2}\right)=\int_{E \times\left\{\theta_{1} \geq \theta_{2}\right\}}\left(\theta_{1}-\theta_{2}\right) \mathrm{d} \mu_{12}\left(x, \theta_{1}, \theta_{2}\right) \geq 0,
\end{aligned}
$$

for every measurable subset $E$ of $\Omega$. This implies $\operatorname{bar}\left(\mu_{1}\right) \geq \operatorname{bar}\left(\mu_{2}\right)$ a.e. in $\Omega$. On the other hand, let us consider

$$
\begin{aligned}
& \mu_{1}^{x}:=\frac{1}{2} \delta_{1 / 4}+\frac{1}{2} \delta_{3 / 4} \text { for a.e. } x \in \Omega \\
& \mu_{2}^{x}:=\frac{1}{2} \delta_{0}+\frac{1}{2} \delta_{1} \text { for a.e. } x \in \Omega .
\end{aligned}
$$

We have $\operatorname{bar}\left(\mu_{1}\right)=\operatorname{bar}\left(\mu_{2}\right) \equiv \frac{1}{2}$ a.e. in $\Omega$, but $\mu_{1}^{x}(0,1]=1>\mu_{2}^{x}(0,1]=1 / 2$ and $\mu_{1}^{x}(3 / 4,1]=$ $0<\mu_{2}^{x}(3 / 4,1]=1 / 2$, for a.e. $x \in \Omega$, therefore $\mu_{1} \nsucceq \mu_{2} \nsucceq \mu_{1}$.

3.2. Sequences of functions generating a Young measure. Let us recall (see [32, Theorem 7.7]) that any Young measure $\mu \in Y^{p}\left(\Omega ; \mathbb{R}^{N}\right)$ can be generated by a suitable sequence of functions $\left(z_{n}\right)_{n} \subset L^{p}\left(\Omega ; \mathbb{R}^{N}\right)$, in the sense that $\boldsymbol{\delta}_{z_{n}} \rightarrow \mu p$-weakly*, as $n \rightarrow \infty$.

In particular, given a measure $\mu_{12} \in Y(\Omega ;[a, b] \times[c, d])$, for $-\infty<a<b<\infty,-\infty<$ $c<d<\infty$, there exists a sequence $\left(z_{n}^{1}, z_{n}^{2}\right)_{n}$ of pairs of functions in $L^{1}(\Omega ;[a, b] \times[c, d])$, such that $\boldsymbol{\delta}_{\left(z_{n}^{1}(x), z_{n}^{2}(x)\right)} \rightarrow \mu_{12}$ weakly*. The question we want to consider in this section is the following: assume that we have already fixed a sequence $\left(\bar{z}_{n}^{1}\right)_{n}$ generating the projection of $\mu_{12}$ over $\Omega \times[a, b]$. Is it possible to construct a sequence $\left(z_{n}^{2}\right)_{n}$ such that $\boldsymbol{\delta}_{\left(\bar{z}_{n}^{1}, z_{n}^{2}\right)} \rightarrow \mu_{12}$ weakly* as $n \rightarrow \infty$ ? An affirmative answer to this question is given by the following.

Theorem 3.8. (Measure reconstruction) Let $\Omega$ be a bounded open subset of $\mathbb{R}^{d}$, and $\mu$ a measure in $Y\left(\Omega ; \mathbb{R}^{2}\right)$ with support contained in $\Omega \times[a, b] \times[c, d]$, for $-\infty<a<b<\infty$, $-\infty<c<d<\infty$. We write $\mu_{1}$ for $\pi_{1}(\mu)$ and $\mu_{2}$ for $\pi_{2}(\mu)$, where $\pi_{1}(x, \theta, \xi):=(x, \theta)$ and $\pi_{2}(x, \theta, \xi):=(x, \xi)$, for every $(x, \theta, \xi) \in \Omega \times \mathbb{R}^{2}$.

Given a sequence $\left(z_{n}^{1}\right)_{n}$ in $L^{\infty}(\Omega ;[a, b])$ such that

$$
\boldsymbol{\delta}_{z_{n}^{1}} \rightarrow \mu_{1} \text { weakly* }
$$

there exists a sequence $\left(z_{n}^{2}\right)_{n}$ in $L^{\infty}(\Omega ;[c, d])$ such that

$$
\boldsymbol{\delta}_{\left(z_{n}^{1}, z_{n}^{2}\right)} \rightarrow \mu \text { weakly* }
$$

Proof. For every $m$, we consider a finite partition of measurable sets $\left(\Omega_{i}^{m}\right)_{i=1}^{I(m)}$ of $\Omega$, and two finite partitions of intervals $\left(H_{j}^{m}\right)_{j=1}^{J(m)}$ of $[a-1, b+1]$ and $\left(K_{k}^{m}\right)_{k=1}^{K(m)}$ of $[c, d]$. We choose these three partitions in such a way that the diameter of each $\Omega_{i}^{m}, H_{j}^{m}$, and $K_{k}^{m}$ is less than $1 / m$. Since the support of $\mu_{1}$ is strictly contained in $[a-1, b+1]$, it is not difficult to see 
that we can always choose $\left(H_{j}^{m}\right)_{j}$ such that $\mu_{1}\left(\Omega_{i}^{m} \times \partial H_{j}^{m}\right)=0$ for every $i=1, \ldots, I(m)$ and $j=1, \ldots J(m)$. Hence, $\mu_{1}\left(\partial\left(\Omega_{i}^{m} \times H_{j}^{m}\right)\right)=0$ for every $i$ and $j$, thanks to the projection property on $\Omega$ satisfied by the Young measure $\mu_{1}$.

We fix now $n \in \mathbb{N}$ and, for every $i=1, \ldots, I(m)$, we define a family of subsets of $\Omega_{i}^{m}$, which we term $\left(\Omega_{i j}^{m, n}\right)_{j=1}^{J(m)}$, by setting

$$
\Omega_{i j}^{m, n}:=\left\{x \in \Omega_{i}^{m}: z_{n}^{1}(x) \in H_{j}^{m}\right\},
$$

for every $j=1, \ldots, J(m)$. Since $\left(H_{j}^{m}\right)_{j}$ are pairwise disjoint, $\left(\Omega_{i j}^{m, n}\right)_{j}$ are pairwise disjoint too, and $\bigcup_{j=1}^{J(m)} \Omega_{i j}^{m, n}=\Omega_{i}^{m}$. We observe that $\sum_{k=1}^{K(m)} \mu\left(\Omega_{i}^{m} \times H_{j}^{m} \times K_{k}^{m}\right)=\mu\left(\bigcup_{k=1}^{K(m)} \Omega_{i}^{m} \times H_{j}^{m} \times\right.$ $\left.K_{k}^{m}\right)=\mu\left(\Omega_{i}^{m} \times H_{j}^{m} \times[c, d]\right)$, hence, if $\mu\left(\Omega_{i}^{m} \times H_{j}^{m} \times[c, d]\right)>0$ we have

$$
\begin{aligned}
\frac{\mu\left(\Omega_{i}^{m} \times H_{j}^{m} \times K_{k}^{m}\right)}{\mu\left(\Omega_{i}^{m} \times H_{j}^{m} \times[c, d]\right)} & \leq 1 \text { for every } k=1, \ldots, K(m), \\
\sum_{k=1}^{K(m)} \frac{\mu\left(\Omega_{i}^{m} \times H_{j}^{m} \times K_{k}^{m}\right)}{\mu\left(\Omega_{i}^{m} \times H_{j}^{m} \times[c, d]\right)} & =1 .
\end{aligned}
$$

Let us set $\mathcal{A}:=\left\{(i, j): \mu\left(\Omega_{i}^{m} \times H_{j}^{m} \times[c, d]\right)=0\right\}$. Therefore, for every $(i, j) \notin \mathcal{A}$, it is possible to find a family of pairwise disjoint subsets of $\Omega_{i j}^{m, n}$, which we denote by $\left(\Omega_{i j k}^{m, n}\right)_{k=1}^{K(m)}$ such that $\bigcup_{k=1}^{K(m)} \Omega_{i j k}^{m, n}=\Omega_{i j}^{m, n}$, and satisfying

$$
\left|\Omega_{i j k}^{m, n}\right|=\frac{\mu\left(\Omega_{i}^{m} \times H_{j}^{m} \times K_{k}^{m}\right)}{\mu\left(\Omega_{i}^{m} \times H_{j}^{m} \times[c, d]\right)}\left|\Omega_{i j}^{m, n}\right| .
$$

Let us define $z_{n}^{m, 2}(x):=\xi_{k}^{m}$, for some $\xi_{k}^{m} \in K_{k}^{m}$, whenever $x \in \Omega_{i j k}^{m, n}$ for $(i, j) \notin \mathcal{A}$, and $z_{n}^{m, 2}(x):=c$ whenever $x \in \Omega_{i j}^{m, n}$ for $(i, j) \in \mathcal{A}$. have

Since $\boldsymbol{\delta}_{z_{n}^{1}} \rightarrow \mu_{1}$, thanks to assumption 3.14 , and $\mu_{1}\left(\partial\left(\Omega_{i}^{m} \times H_{j}^{m}\right)\right)=0$ for every $i, j$, we

$$
\left|\Omega_{i j}^{m, n}\right|=\boldsymbol{\delta}_{z_{n}^{1}}\left(\Omega_{i}^{m} \times H_{j}^{m}\right) \longrightarrow \mu_{1}\left(\Omega_{i}^{m} \times H_{j}^{m}\right)=\mu\left(\Omega_{i}^{m} \times H_{j}^{m} \times[c, d]\right),
$$

as $n \rightarrow \infty$. Therefore, for every $m$, there exists $n_{m}$ such that

$$
\begin{aligned}
\left|\frac{\left|\Omega_{i j}^{m, n}\right|}{\mu\left(\Omega_{i}^{m} \times H_{j}^{m} \times[c, d]\right)}-1\right| & \leq \frac{1}{m} \text { for every }(i, j) \notin \mathcal{A}, \\
\sum_{(i, j) \in \mathcal{A}}\left|\Omega_{i, j}^{m, n}\right| & \leq \frac{1}{m} .
\end{aligned}
$$

whenever $n \geq n_{m}$. Without loss of generality we can assume that $\left(n_{m}\right)_{m}$ is an increasing sequence of integers. We are now ready to define $z_{n}^{2}$, by setting $z_{n}^{2}(x):=z_{n}^{m, 2}(x)$ whenever $n_{m} \leq n<n_{m+1}$. 
We have now to show that for every $f \in C_{0}\left(\Omega \times \mathbb{R}^{2}\right)$ and for every $\varepsilon>0$ there exists $N$ such that

$$
\left|\int_{\Omega \times \mathbb{R}^{2}} f(x, \theta, \xi) \mathrm{d} \boldsymbol{\delta}_{\left(z_{n}^{1}(x), z_{n}^{2}(x)\right)}-\int_{\Omega \times \mathbb{R}^{2}} f(x, \theta, \xi) \mathrm{d} \mu(x, \theta, \xi)\right| \leq \varepsilon
$$

whenever $n \geq N$.

Since $f$ is uniformly continuous, there exists a modulus of continuity $\omega_{f}: \mathbb{R} \rightarrow \mathbb{R}$, namely, for every $\left(x_{1}, \theta_{1}, \xi_{1}\right),\left(x_{2}, \theta_{2}, \xi_{2}\right) \in \Omega \times \mathbb{R}^{2}$,

$$
\left|f\left(x_{1}, \theta_{1}, \xi_{1}\right)-f\left(x_{2}, \theta_{2}, \xi_{2}\right)\right| \leq \omega_{f}\left(\left|\left(x_{1}, \theta_{1}, \xi_{1}\right)-\left(x_{2}, \theta_{2}, \xi_{2}\right)\right|\right), \quad \text { with } \lim _{\delta \rightarrow 0} \omega_{f}(\delta)=0
$$

Given $n$, let $m$ be such that $n_{m} \leq n<n_{m+1}$. Then we have

$$
\begin{aligned}
& \int_{\Omega \times \mathbb{R}^{2}} f(x, \theta, \xi) \mathrm{d} \boldsymbol{\delta}_{\left(z_{n}^{1}, z_{n}^{2}\right)}(x, \theta, \xi)=\int_{\Omega \times \mathbb{R}^{2}} f(x, \theta, \xi) \mathrm{d} \boldsymbol{\delta}_{\left(z_{n}^{1}, z_{n}^{m, 2}\right)}(x, \theta, \xi) \\
= & \sum_{(i, j) \notin \mathcal{A}} \sum_{k} \int_{\Omega_{i j k}^{m, n}} f\left(x, z_{n}^{1}(x), \xi_{k}^{m}\right) \mathrm{d} x+\sum_{(i, j) \in \mathcal{A}} \int_{\Omega_{i, j}^{m, n}} f\left(x, z_{n}^{1}(x), c\right) \mathrm{d} x .
\end{aligned}
$$

In particular, for every $x_{i}^{m} \in \Omega_{i}^{m}$ and $\theta_{j}^{m} \in H_{j}^{m}$ we have

$$
\begin{aligned}
& \left|\int_{\Omega \times \mathbb{R}^{2}} f(x, \theta, \xi) \mathrm{d} \boldsymbol{\delta}_{\left(z_{n}^{1}, z_{n}^{2}\right)}(x, \theta, \xi)-\sum_{(i, j) \notin \mathcal{A}} \sum_{k} \int_{\Omega_{i j k}^{m, n}} f\left(x_{i}^{m}, \theta_{j}^{m}, \xi_{k}^{m}\right) \mathrm{d} x-\sum_{(i, j) \in \mathcal{A}} \int_{\Omega_{i, j}^{m, n}} f\left(x_{i}^{m}, \theta_{j}^{m}, c\right) \mathrm{d} x\right| \\
\leq & \sum_{(i, j) \notin \mathcal{A}} \sum_{k} \int_{\Omega_{i j k}^{m, n}}\left|f\left(x, z_{n}^{1}(x), \xi_{k}^{m}\right)-f\left(x_{i}^{m}, \theta_{j}^{m}, \xi_{k}^{m}\right)\right| \mathrm{d} x+\sum_{(i, j) \in \mathcal{A}} \int_{\Omega_{i, j}^{m, n}}\left|f\left(x, z_{n}^{1}(x), c\right)-f\left(x_{i}^{m}, \theta_{j}^{m}, c\right)\right| \mathrm{d} x \\
\leq & \omega_{f}(2 / m)\left[\sum_{(i, j) \notin \mathcal{A}} \sum_{k}\left|\Omega_{i j k}^{m, n}\right|+\sum_{(i, j) \in \mathcal{A}}\left|\Omega_{i, j}^{m, n}\right|\right]=\omega_{f}(2 / m) \sum_{i j}\left|\Omega_{i j}^{m, n}\right| \\
= & \omega_{f}(2 / m) \sum_{i}\left|\Omega_{i}^{m}\right|=\omega_{f}(2 / m)|\Omega| .
\end{aligned}
$$


Using now the construction of $\Omega_{i j k}^{m, n}$, and the estimates 3.15 and 3.16 , we get

$$
\begin{aligned}
& \mid \sum_{(i, j) \notin \mathcal{A}} \sum_{k} \int_{\Omega_{i j k}^{m, n}} f\left(x_{i}^{m}, \theta_{j}^{m}, \xi_{k}^{m}\right) \mathrm{d} x+\sum_{(i, j) \in \mathcal{A}} \int_{\Omega_{i, j}^{m, n}} f\left(x_{i}^{m}, \theta_{j}^{m}, c\right) \mathrm{d} x \\
& -\sum_{(i, j) \notin \mathcal{A}} \sum_{k} f\left(x_{i}^{m}, \theta_{j}^{m}, \xi_{k}^{m}\right) \mu\left(\Omega_{i}^{m} \times H_{j}^{m} \times K_{k}^{m}\right)-\sum_{(i, j) \in \mathcal{A}} f\left(x_{i}^{m}, \theta_{j}^{m}, c\right) \mu\left(\Omega_{i}^{m} \times H_{j}^{m} \times[c, d]\right) \mid \\
\leq & \left|\sum_{(i, j) \notin \mathcal{A}} \sum_{k} f\left(x_{i}^{m}, \theta_{j}^{m}, \xi_{k}^{m}\right)\right| \Omega_{i j k}^{m, n}\left|-\sum_{(i, j) \notin \mathcal{A}} \sum_{k} f\left(x_{i}^{m}, \theta_{j}^{m}, \xi_{k}^{m}\right) \mu\left(\Omega_{i}^{m} \times H_{j}^{m} \times K_{k}^{m}\right)\right| \\
& +\left|\sum_{(i, j) \in \mathcal{A}} f\left(x_{i}^{m}, \theta_{j}^{m}, c\right)\right| \Omega_{i, j}^{m, n}|-0| \\
\leq & \|f\|_{\infty} \sum_{(i, j) \notin \mathcal{A}} \sum_{k} \mu\left(\Omega_{i}^{m} \times H_{j}^{m} \times K_{k}^{m}\right)\left[\frac{\left|\Omega_{i j}^{m, n}\right|}{\mu\left(\Omega_{i}^{m} \times H_{j}^{m} \times[c, d]\right)}-1\right]+\|f\|_{\infty} \sum_{(i, j) \in \mathcal{A}}\left|\Omega_{i, j}^{m, n}\right| \\
= & \frac{\|f\|_{\infty}}{m}\left[\sum_{i j} \mu\left(\Omega_{i}^{m} \times H_{j}^{m} \times[c, d]\right)+1\right]=\frac{\|f\|_{\infty}}{m}[|\Omega|+1] .
\end{aligned}
$$

Finally, we have

$$
\begin{aligned}
& \mid \sum_{(i, j) \notin \mathcal{A}} \sum_{k} f\left(x_{i}^{m}, \theta_{j}^{m}, \xi_{k}^{m}\right) \mu\left(\Omega_{i}^{m} \times H_{j}^{m} \times K_{k}^{m}\right)+\sum_{(i, j) \in \mathcal{A}} f\left(x_{i}^{m}, \theta_{j}^{m}, c\right) \mu\left(\Omega_{i}^{m} \times H_{j}^{m} \times[c, d]\right) \\
& -\int_{\Omega \times \mathbb{R}^{2}} f(x, \theta, \xi) \mathrm{d} \mu(x, \theta, \xi) \mid \\
\leq & \sum_{(i, j) \notin \mathcal{A}} \sum_{k} \int_{\Omega_{i}^{m} \times H_{j}^{m} \times K_{k}^{m}}\left|f\left(x_{i}^{m}, \theta_{j}^{m}, \xi_{k}^{m}\right)-f(x, \theta, \xi)\right| \mathrm{d} \mu(x, \theta, \xi) \\
& +\sum_{(i, j) \in \mathcal{A}} \int_{\Omega_{i}^{m} \times H_{j}^{m} \times[c, d]}|f(x, \theta, \xi)| \mathrm{d} \mu(x, \theta, \xi) \\
\leq & \omega_{f}(3 / m) \sum_{(i, j) \notin \mathcal{A}} \sum_{k} \mu\left(\Omega_{i}^{m} \times H_{j}^{m} \times K_{k}^{m}\right)+\|f\|_{\infty} \sum_{(i, j) \in \mathcal{A}} \mu\left(\Omega_{i}^{m} \times H_{j}^{m} \times[c, d]\right) \\
= & \omega_{f}(3 / m)|\Omega| .
\end{aligned}
$$

Therefore, putting together the estimates (3.18), 3.19), and (3.20) we obtain

$$
\begin{gathered}
\left|\int_{\Omega \times \mathbb{R}^{2}} f(x, \theta, \xi) \mathrm{d} \boldsymbol{\delta}_{\left(z_{n}^{1}, z_{n}^{m, 2}\right)}(x, \theta, \xi)-\int_{\Omega \times \mathbb{R}^{2}} f(x, \theta, \xi) \mathrm{d} \mu(x, \theta, \xi)\right| \\
\leq|\Omega|\left[\omega_{f}\left(\frac{2}{m}\right)+\frac{\|f\|_{\infty}}{m}+\omega_{f}\left(\frac{3}{m}\right)\right]+\frac{\|f\|_{\infty}}{m} .
\end{gathered}
$$

In particular, fixed $\varepsilon>0$, condition (3.17) is satisfied for $m$ sufficiently large, $m \geq M$. Hence it is enough to choose $N$ such that $n_{M} \leq N \leq n_{M+1}$. In this way, for every $n \geq N$, we have $n_{m} \leq n<n_{m+1}$ for some $m \geq M$ and hence (3.17) holds true for every $n \geq N$. 
Corollary 3.9. (Measure reconstruction with order) In addition to the hypotheses of Theorem 3.8, if $a=c, b=d$, and $\mu$ satisfies the condition

$$
\mu^{x}\left(\left\{(\theta, \xi) \in \mathbb{R}^{2}: \theta<\xi\right\}\right)=0 \text { for a.e. } x \in \Omega,
$$

we can construct the sequence $\left(z_{n}^{2}\right)_{n}$ with the property

$$
z_{n}^{1}(x) \geq z_{n}^{2}(x) \quad \text { for a.e. } x \in \Omega
$$

Proof. For every $m$, we can assume that $\left(H_{j}^{m}\right)_{j}$ is ordered in the sense that $\theta_{j+1}>\theta_{j}$ whenever $\theta_{j} \in H_{j}^{m}, \theta_{j+1} \in H_{j+1}^{m}$. Since $[c, d]=[a, b]$, we can choose $K_{k}^{m}:=H_{k}^{m} \cap[a, b]$ for every $k$. If $(i, j) \in \mathcal{A}, z_{n}^{2, m}(x)=a \leq z_{n}^{1}(x)$ for almost every $x \in \Omega_{i, j}^{m, n}$. So let us consider from now on $(i, j) \notin \mathcal{A}$. Since $\mu^{x}(\{\theta<\xi\})=0$ for almost every $x \in \Omega$, due to assumption (3.21), we have that $\mu\left(\Omega_{i}^{m} \times H_{j}^{m} \times\left(H_{k}^{m} \cap[a, b]\right)\right)=0$ for every $i$ and every $k>j$. In particular, $\left|\Omega_{i j k}^{m, n}\right|=0$ whenever $k>j$. Nothing changes in the proof of Theorem 3.8 if we take $\xi_{k}^{m}$ in the closure of $K_{k}^{m} \cap[a, b]$. In this way, for $k=j$ we are able to choose $\xi_{j}^{m}$ with the property $\xi_{j}^{m} \leq z_{n}^{1}(x)$ whenever $x \in \Omega_{i j}^{m, n}$ (notice that $z_{n}^{1}(x) \in[a, b]$ ), so that $z_{n}^{2, m}(x) \leq z_{n}^{1}(x)$ whenever $x \in \Omega_{i j j}^{m, n}$. Finally, if $k<j$, then for every $\xi_{k}^{m} \in H_{k}^{m} \cap[a, b]$ we have $\xi_{k}^{m} \leq z_{n}^{1}(x)$ whenever $x \in \Omega_{i j}^{m, n}$ and hence $z_{n}^{2, m}(x) \leq z_{n}^{1}(x)$ whenever $x \in \Omega_{i j k}^{m, n}$. In conclusion, $z_{n}^{2, m}(x) \leq z_{n}^{1}(x)$ for almost every $x \in \Omega$, and hence $z_{n}^{2}(x) \leq z_{n}^{1}(x)$ for almost every $x \in \Omega$ too.

3.3. Admissible set in terms of Young measures. We now introduce the admissible set for the generalized notion of evolution we will consider.We recall that $\mu \in Y^{2}\left(\Omega ; \mathbb{R}^{d \times d}\right)$ is a $H^{1}$-gradient Young measure $\left(H^{1}\right.$-GYM), if there exists a bounded sequence $\left(v_{n}\right)_{n} \in H^{1}\left(\Omega ; \mathbb{R}^{d}\right)$ such that $\boldsymbol{\delta}_{\nabla v_{n}} \rightarrow \mu 2$-weakly* as $n \rightarrow \infty$.

Definition 3.10. (Admissible set) Given a set of times $[0, T]$ and $\varphi:[0, T] \rightarrow W^{1, p}\left(\Omega ; \mathbb{R}^{d}\right)$, for $p>2$, we define $A Y([0, T], \varphi)$ as the set of all $\nu \in Y^{2}\left(\Omega ; \mathbb{R} \times \mathbb{R}_{\mathrm{sym}}^{d \times d}\right)^{[0, T]}$ such that for every $t \in[0, T]$ there exists a measure $\tilde{\nu}_{t} \in Y^{2}\left(\Omega ; \mathbb{R} \times \mathbb{R}^{d \times d}\right)$ with

$$
\begin{aligned}
& \nu_{t}=S \tilde{\nu}_{t}, \\
& \operatorname{supp} \pi_{1}\left(\tilde{\nu}_{t}\right)=\operatorname{supp} \pi_{1}\left(\nu_{t}\right) \subseteq \Omega \times[0,1] \\
& \pi_{2}\left(\tilde{\nu}_{t}\right) \text { is a } H^{1}-\mathrm{GYM} \text {, and } \operatorname{bar}\left(\pi_{2} \tilde{\nu}_{t}\right)=\nabla v \text { with } v \in \varphi(t)+H_{0}^{1}\left(\Omega ; \mathbb{R}^{d}\right),
\end{aligned}
$$

where $S(x, \theta, F):=\left(x, \theta, \frac{F+F^{T}}{2}\right)$ for every $(x, \theta, F) \in \Omega \times \mathbb{R} \times \mathbb{R}^{d \times d}$, and $\pi_{1}$ and $\pi_{2}$ are projections, $\pi_{1}: \Omega \times \mathbb{R} \times \mathbb{R}^{d \times d} \rightarrow \Omega \times \mathbb{R}$ and $\pi_{2}: \Omega \rightarrow \mathbb{R} \times \mathbb{R}^{d \times d} \rightarrow \Omega \times \mathbb{R}^{d \times d}$, respectively.

From [13, Theorem 3.1], $\tilde{\nu}_{t}$ satisfies properties (3.23) and (3.24) if and only if there exist a bounded sequence $\left(z_{n}\right)_{n}$ in $L^{\infty}(\Omega ;[0,1])$ and a bounded sequence $\left(v_{n}\right)_{n}$ in $H^{1}\left(\Omega ; \mathbb{R}^{d \times d}\right)$ such that $\boldsymbol{\delta}_{\left(z_{n}, \nabla v_{n}\right)} \rightarrow \tilde{\nu}_{t} 2$-weakly* as $n \rightarrow \infty$. Moreover, by using for instance [1, Lemma 11.4.1], it is possible to choose $\left(v_{n}\right)_{n}$ in $\varphi(t)+H_{0}^{1}\left(\Omega ; \mathbb{R}^{d \times d}\right)$. Eventually note that $\boldsymbol{\delta}_{\left(z_{n}, \nabla v_{n}\right)} \rightarrow \tilde{\nu}_{t}$ 2-weakly* implies $\boldsymbol{\delta}_{\left(z_{n}, e\left(v_{n}\right)\right)} \rightarrow S \tilde{\nu}_{t} 2$-weakly*. 


\section{MAIN RESUlT}

We shall now aim at introducing the existence result for quasi-static damage evolution.

Before giving the definition of quasi-static damage evolution and stating the main result, we need to be fixing some extra notation.

Given $\nu \in Y^{2}\left(\Omega ; \mathbb{R} \times \mathbb{R}_{\mathrm{sym}}^{d \times d}\right)$ and $\mu_{12} \in Y^{1}\left(\Omega ;[0,1]^{2}\right)$, we set

$$
\begin{aligned}
\langle W, \nu\rangle: & =\int_{\Omega \times \mathbb{R} \times \mathbb{R}_{\mathrm{sym}}^{d \times d}} W(\theta, \varepsilon) \mathrm{d} \nu(x, \theta, \varepsilon), \\
\left\langle d, \mu_{12}\right\rangle: & =\int_{\Omega \times \mathbb{R}^{2}} d\left(\theta_{1}, \theta_{2}\right) \mathrm{d} \mu_{12}\left(x, \theta_{1}, \theta_{2}\right) .
\end{aligned}
$$

Given $\mu_{1}, \mu_{2} \in Y^{1}(\Omega ;[0,1])$, we define

$$
\mathbb{D}\left(\mu_{1}, \mu_{2}\right):= \begin{cases}\rho\left[\int_{\Omega \times \mathbb{R}} \theta \mathrm{d} \mu_{1}(x, \theta)-\int_{\Omega \times \mathbb{R}} \theta \mathrm{d} \mu_{2}(x, \theta)\right] & \text { if } \mu_{1} \succeq \mu_{2} \\ \infty & \text { otherwise. }\end{cases}
$$

The distance $\mathbb{D}\left(\mu_{1}, \mu_{2}\right)$ coincides with the infimum of $\left\langle d, \mu_{12}\right\rangle$ for $\mu_{12}$ varying in the set of measures in $Y^{1}\left(\Omega ;[0,1]^{2}\right)$ such that $\pi_{1}\left(\mu_{12}\right)=\mu_{1}$ and $\pi_{2}\left(\mu_{12}\right)=\mu_{2}$, where $\pi_{1}\left(x, \theta_{1}, \theta_{2}\right):=$ $\left(x, \theta_{1}\right)$ and $\pi_{2}\left(x, \theta_{1}, \theta_{2}\right):=\left(x, \theta_{2}\right)$ for every $\left(x, \theta_{1}, \theta_{2}\right) \in \Omega \times \mathbb{R}^{2}$. Indeed, this is true if $\mu_{1} \nsucceq \mu_{2}$, because, thanks to Theorem 3.6 and to the definition of $d$, in this case we have $\left\langle d, \mu_{12}\right\rangle=\infty$ for every $\mu_{12}$ satisfying the required projection properties. On the other hand, if $\mu_{1} \succeq \mu_{2}$, by Theorem 3.6 there exists a measure $\mu_{12}$ satisfying the projection properties and with $\mu_{12}^{x}\left(\left\{\theta_{1}<\right.\right.$ $\left.\left.\theta_{2}\right\}\right)=0$ for almost every $x \in \Omega$. Therefore, for every such a measure $\mu_{12}$, we have $\left\langle d, \mu_{12}\right\rangle<\infty$ and

$$
\begin{gathered}
\left\langle d, \mu_{12}\right\rangle=\int_{\Omega \times[0,1]^{2}} d\left(\theta_{1}, \theta_{2}\right) \mathrm{d} \mu_{12}\left(x, \theta_{1}, \theta_{2}\right)=\int_{\Omega}\left(\int_{\left\{\theta_{1} \geq \theta_{2}\right\}} d\left(\theta_{1}, \theta_{2}\right) \mathrm{d} \mu_{12}^{x}\left(\theta_{1}, \theta_{2}\right)\right) \mathrm{d} x \\
=\int_{\Omega}\left(\int_{\left\{\theta_{1} \geq \theta_{2}\right\}} \rho\left(\theta_{1}-\theta_{2}\right) \mathrm{d} \mu_{12}^{x}\left(\theta_{1}, \theta_{2}\right)\right) \mathrm{d} x=\rho \int_{\Omega \times[0,1]^{2}}\left(\theta_{1}-\theta_{2}\right) \mathrm{d} \mu_{12}\left(x, \theta_{1}, \theta_{2}\right) \\
=\rho\left[\int_{\Omega \times \mathbb{R}} \theta_{1} \mathrm{~d} \mu_{1}\left(x, \theta_{1}\right)-\int_{\Omega \times \mathbb{R}} \theta_{2} \mathrm{~d} \mu_{2}\left(x, \theta_{2}\right)\right] .
\end{gathered}
$$

Therefore, $\left\langle d, \mu_{12}\right\rangle$ is independent of the choice of $\mu_{12}$, provided it has the required order property, and coincides with $\mathbb{D}\left(\mu_{1}, \mu_{2}\right)$. In other words, $\mathbb{D}\left(\mu_{1}, \mu_{2}\right)$ corresponds to a Wassersteinlike distance associated with $d$ between $\mu_{1}$ and $\mu_{2}$ (see e.g. [25]). Note that we may have $\mathbb{D}\left(\mu_{1}, \mu_{2}\right)=\infty$ and $\mathcal{D}\left(\operatorname{bar}\left(\mu_{1}\right), \operatorname{bar}\left(\mu_{2}\right)\right)<\infty$, because $\mu_{1} \succeq \mu_{2}$ is a stronger condition than $\operatorname{bar}\left(\mu_{1}\right) \geq \operatorname{bar}\left(\mu_{2}\right)$ almost everywhere in $\Omega$, as explained in Remark 3.7 .

Given a measure $\nu \in Y^{2}\left(\Omega ;[0,1] \times \mathbb{R}^{d \times d}\right)$, we will denote the projection of $\nu$ on $\Omega \times[0,1]$ by $\pi_{1}(\nu)$. We are now ready to define our solution notion for the quasi-static problem.

Definition 4.1. (Quasi-static evolution) Given $\varphi:[0, T] \times \Omega \rightarrow \mathbb{R}^{d}, z_{0}: \Omega \rightarrow[0,1], v_{0}: \Omega \rightarrow$ $\mathbb{R}^{d}$, and $T>0$, a quasi-static damage evolution with boundary datum $\varphi$ and initial condition $\left(z_{0}, v_{0}\right)$, in the time interval $[0, T]$, is $\nu \in A Y([0, T], \varphi)$, satisfying the following conditions: 
(E0) initial condition: $\nu_{0}=\delta_{\left(z_{0}, e\left(v_{0}\right)\right)}$,

(E1) irreversibility: $\pi_{1}\left(\nu_{s}\right) \succeq \pi_{1}\left(\nu_{t}\right)$, whenever $0 \leq s<t \leq T$,

(E2) translational stability: for every $t \in[0, T]$, we have

$$
\left\langle W, \nu_{t}\right\rangle \leq\left\langle W, \operatorname{Tr}_{(\tilde{z}, e(\tilde{u}))}\left(\nu_{t}\right)\right\rangle+\mathbb{D}\left(\pi_{1} \nu_{t}, \operatorname{Tr}_{\tilde{z}}\left(\pi_{1} \nu_{t}\right)\right),
$$

for every $\tilde{z} \in L^{1}(\Omega)$ and every $\tilde{u} \in H_{0}^{1}\left(\Omega ; \mathbb{R}^{d}\right)$,

(E3) global-stability for the internal variable: for every $t \in[0, T]$, we have

$$
\left\langle W, \nu_{t}\right\rangle \leq\left\langle W,\left(\tilde{\mu}^{x} \otimes \delta_{e(\tilde{v})(x)}\right)_{x \in \Omega}\right\rangle+\mathbb{D}\left(\pi_{1}\left(\nu_{t}\right), \tilde{\mu}\right),
$$

for every $\tilde{v} \in \varphi(t)+H_{0}^{1}\left(\Omega ; \mathbb{R}^{d}\right)$, and every $\tilde{\mu} \in Y(\Omega ; \mathbb{R})$,

(E4) energy equality: for every $t \in[0, T]$ the map

$$
t \mapsto\langle\sigma(t), e(\dot{\varphi}(t))\rangle
$$

is measurable on $[0, T]$, where $\sigma(t)$ is the function defined by

$$
\sigma(t)(x):=\int_{\mathbb{R} \times \mathbb{R}_{\mathrm{sym}}^{d \times d}} \frac{\partial W}{\partial \varepsilon}(\theta, \varepsilon) \mathrm{d} \nu_{t}^{x}(\theta, \varepsilon) ;
$$

moreover for every $t \in[0, T]$ we have

$$
\left\langle W, \nu_{t}\right\rangle+\operatorname{Diss}(\nu ; 0, t)=\mathcal{W}\left(z_{0}, v_{0}\right)+\int_{0}^{t}\langle\sigma(s), e(\dot{\varphi}(s))\rangle \mathrm{d} s,
$$

where $\operatorname{Diss}(\nu ; 0, t):=\sup \sum_{i=1}^{k} \mathbb{D}\left(\pi_{1} \nu_{t_{i-1}}, \pi_{1} \nu_{t_{i}}\right)$, where the supremum is taken among all finite partitions $0=t_{0}<\cdots<t_{k}=t$.

The main result of this paper reads as follows.

Theorem 4.2. (Existence of a quasi-static evolution) Let $\varphi \in A C\left([0, T] ; W^{1, p}\left(\Omega ; \mathbb{R}^{d}\right)\right)$, $p>2, T>0, z_{0} \in L^{1}(\Omega ;[0,1])$, and $v_{0} \in \varphi(0)+H_{0}^{1}\left(\Omega ; \mathbb{R}^{d}\right)$ be such that

$$
\mathcal{W}\left(z_{0}, v_{0}\right) \leq \mathcal{W}(\tilde{z}, \tilde{v})+\mathcal{D}\left(z_{0}, \tilde{z}\right)
$$

for every $\tilde{z} \in L^{1}(\Omega)$ and every $\tilde{v} \in \varphi(0)+H_{0}^{1}\left(\Omega ; \mathbb{R}^{d}\right)$. Then there exists a quasi-static evolution with boundary datum $\varphi$ and initial condition $\left(z_{0}, v_{0}\right)$ in the time interval $[0, T]$.

The proof is obtained via time discretization, incremental minimization, and passage to the limit and is detailed in Section 5 .

\section{Proof of the EXISTence theorem 4.2}

5.1. The incremental minimum problem. Let us fix a time step $\tau:=T / n$, and let $t_{\tau}^{i}:=i \tau$ and $\varphi_{\tau}^{i}:=\varphi\left(t_{\tau}^{i}\right)$, for every $i=0, \ldots, n$. We will define $\left(z_{\tau}^{i}, v_{\tau}^{i}\right)$ iteratively: set $\left(z_{\tau}^{0}, v_{\tau}^{0}\right):=$ $\left(z_{0}, v_{0}\right)$, and, for $i>0$, define $\left(z_{\tau}^{i}, v_{\tau}^{i}\right)$ as a minimizer (see Lemma 5.1 below) of the functional

$$
\mathcal{F}_{\tau}^{i}(z, v):=\mathcal{W}(z, e(v))+\mathcal{D}\left(z_{\tau}^{i-1}, z\right),
$$

among all $z \in L^{1}(\Omega)$ and $v \in \varphi_{\tau}^{i}+H_{0}^{1}\left(\Omega ; \mathbb{R}^{d}\right)$. 
Lemma 5.1. (Incremental minimization) Let $\left(z_{0}, v_{0}\right)$ be as in Theorem 4.2. Then, for every $i$ the functional $\mathcal{F}_{\tau}^{i}$ has a minimizer $(z, v)$ in $L^{1}(\Omega) \times\left(\varphi_{\tau}^{i}+H_{0}^{1}\left(\Omega ; \mathbb{R}^{d}\right)\right)$. Moreover, $(z, v)$ satisfies the following properties:

$$
\begin{aligned}
& 0 \leq z \leq z_{\tau}^{i-1} \text { a.e. in } \Omega \\
& v \text { is a } \frac{C_{W}}{c_{W}} \text {-cubic quasi-minimum of the functional } v \mapsto \int_{\Omega}|e(v)|^{2} .
\end{aligned}
$$

Remark 5.2. In particular for every $i$ and $\tau$, we have that $0 \leq z_{\tau}^{i}(x) \leq z_{0}(x) \leq 1$ for almost every $x \in \Omega$, since $z_{0}(x) \in[0,1]$ for almost every $x \in \Omega$.

Proof. Let us first observe that, whenever $z_{\tau}^{i-1} \geq 0$ almost everywhere in $\Omega$, we have

$$
\mathcal{F}_{\tau}^{i}(z, v) \geq \mathcal{F}_{\tau}^{i}\left(\left(z \wedge z_{\tau}^{i-1}\right)^{+}, v\right)
$$

for every $(z, v) \in L^{1}(\Omega) \times H^{1}\left(\Omega ; \mathbb{R}^{d}\right)$. Indeed, $\mathcal{F}_{\tau}^{i}(z, v)<\infty$ if and only if $z \leq z_{\tau}^{i-1}$ almost everywhere in $\Omega$, hence $\mathcal{F}_{\tau}^{i}(z, v) \geq \mathcal{F}_{\tau}^{i}\left(z \wedge z_{\tau}^{i-1}, v\right)$. On the other hand, $W(\theta, \varepsilon) \equiv W(0, \varepsilon)$ if $\theta \leq 0$ (see hypothesis $(W .5)$ ). Hence, $\mathcal{W}\left(z \wedge z_{\tau}^{i-1}, e(v)\right)=\mathcal{W}\left(\left(z \wedge z_{\tau}^{i-1}\right)^{+}, e(v)\right)$. Finally, since $z_{\tau}^{i-1} \geq 0$ almost everywhere in $\Omega, \mathcal{D}\left(z_{\tau}^{i-1},\left(z \wedge z_{\tau}^{i-1}\right)^{+}\right) \leq \mathcal{D}\left(z_{\tau}^{i-1}, z \wedge z_{\tau}^{i-1}\right)$ (with the strict inequality if $z(x) \notin[0,1]$ for almost every $x \in \Omega)$. In conclusion, $\mathcal{F}_{\tau}^{i}(z, v) \geq \mathcal{F}_{\tau}^{i}\left(z \wedge z_{\tau}^{i-1}, v\right) \geq$ $\mathcal{F}_{\tau}^{i}\left(\left(z \wedge z_{\tau}^{i-1}\right)^{+}, v\right)$. This implies that, if $z_{\tau}^{i}$ exists, it satisfies

$$
0 \leq z_{\tau}^{i}(x) \leq z_{\tau}^{i-1}(x) \text { for a.e. } x \in \Omega,
$$

whenever $z_{\tau}^{i-1} \geq 0$ almost everywhere in $\Omega$. Since $z_{0}(x) \in[0,1]$ for a.e. $x \in \Omega$, by induction we get that, if $z_{\tau}^{i}$ exists, it fulfills 5.5 .

Fix now $i=1, \ldots, n$, and let $\left(z_{k}, v_{k}\right)$ be a minimizing sequence for $\mathcal{F}_{\tau}^{i}$. Then

$$
\mathcal{F}_{\tau}^{i}\left(z_{k}, v_{k}\right)=\int_{\Omega} W\left(z_{k}, e\left(v_{k}\right)\right) \mathrm{d} x+\mathcal{D}\left(z_{\tau}^{i-1}, z_{k}\right)<C,
$$

for a suitable positive constant $C$. In particular, thanks to (W.2) and Korn-Poincaré inequality $(3.1)$, the sequence $\left(v_{k}\right)_{k}$ is bounded in $\varphi_{\tau}^{i}+H_{0}^{1}\left(\Omega ; \mathbb{R}^{d}\right)$. Since $z_{\tau}^{i-1} \in L^{1}(\Omega ;[0,1])$, we can apply (5.4) in order to deduce that $\left(\left(z_{k} \wedge z_{\tau}^{i-1}\right)^{+}, v_{k}\right)$ is still a minimizing sequence. Since $z_{\tau}^{i-1} \leq 1$, $\left(z_{k} \wedge z_{\tau}^{i-1}\right)^{+}$is bounded in $L^{\infty}(\Omega)$. Up to a subsequence, we can assume that $v_{k}$ converges weakly in $H^{1}$ to a function $v \in \varphi_{\tau}^{i}+H_{0}^{1}\left(\Omega ; \mathbb{R}^{d}\right)$, and $\left(z_{k} \wedge z_{\tau}^{i-1}\right)^{+}$converges weakly* in $L^{\infty}$ to a function $z$ with values in $[0,1]$ almost everywhere in $\Omega$. Since $W$ is S-cross-quasiconvex, thanks to Lemma 3.3 , the functional in (5.1) is sequentially lower semicontinuous with respect to the product of the weak* topology of $L^{\infty}$ and the weak topology of $H^{1}$. This proves that $(z, v)$ is a minimum of the functional (5.1) and satisfies the condition (5.2).

Hence, it remains to show that $v$ is a $\frac{C_{W}}{c_{W}}$-cubic quasi-minimum of the functional $v \mapsto$ $\int_{\Omega}|e(v)|^{2}$. Let $w$ be a function such that $v-w \in H_{0}^{1}\left(\Omega \cap Q_{R}\right)$. We extend it to a function in $H^{1}\left(\Omega ; \mathbb{R}^{d}\right)$ by setting $w:=v$ on $\Omega \backslash Q_{R}$. Then $(z, w)$ is a competitor for the minimum problem solved by $(z, v)$. Hence,

$$
\int_{\Omega} W(z(x), e(v)(x)) \mathrm{d} x \leq \int_{\Omega} W(z(x), e(w)(x)) \mathrm{d} x .
$$


By construction of $w$, this implies

$$
\int_{\Omega \cap Q_{R}} W(z(x), e(v)(x)) \mathrm{d} x \leq \int_{\Omega \cap Q_{R}} W(z(x), e(w)(x)) \mathrm{d} x .
$$

Hence, by the hypothesis $(W .2)$ on $W$, we get

$$
c_{W} \int_{\Omega \cap Q_{R}}|e(v)(x)|^{2} \mathrm{~d} x \leq C_{W} \int_{\Omega \cap Q_{R}}|e(w)(x)|^{2} \mathrm{~d} x,
$$

which proves that $v$ satisfies the condition (5.3).

Let $\left(z_{\tau}, v_{\tau}\right)$ and $\varphi_{\tau}$ be the functions in $L^{\infty}\left([0, T] ; L^{1}(\Omega) \times H^{1}\left(\Omega ; \mathbb{R}^{d}\right)\right)$ and $L^{\infty}\left([0, T] ; H^{1}\left(\Omega ; \mathbb{R}^{d}\right)\right)$, respectively, defined by

$$
\begin{gathered}
\left(z_{\tau}(t), v_{\tau}(t)\right):=\left(z_{\tau}^{i}, v_{\tau}^{i}\right) \quad \text { if } t_{\tau}^{i} \leq t<t_{\tau}^{i+1}, \\
\varphi_{\tau}(t):=\varphi_{\tau}^{i} \quad \text { if } t_{\tau}^{i} \leq t<t_{\tau}^{i+1}, \quad i=0,1, \ldots, n .
\end{gathered}
$$

We define $\sigma_{\tau} \in L^{\infty}\left([0, T] ; L^{2}\left(\Omega ; \mathbb{R}_{\mathrm{sym}}^{d \times d}\right)\right)$ (thanks to $\left.(W .3)\right)$ by

$$
\sigma_{\tau}(t):=\frac{\partial W}{\partial \varepsilon}\left(z_{\tau}(t), e\left(v_{\tau}(t)\right)\right)
$$

for every $t \in[0, T]$.

5.2. Improved integrability. Since $v_{\tau}^{i}$ is a $\frac{C_{W}}{c_{W}}$-cubic quasi-minimum of the functional $v \mapsto$ $\int_{\Omega}|e(v)|^{2} \mathrm{~d} x$, we use Theorem 3.2 (see also [12, Appendix]) to obtain the existence of two constants $\gamma>0$ and $r>1$, depending only on $c_{W}, C_{W}$, and $\varphi$, such that

$$
\begin{aligned}
\int_{\Omega}\left|e\left(v_{\tau}^{i}\right)\right|^{2 r} \mathrm{~d} x & \leq \int_{\Omega}\left|\nabla v_{\tau}^{i}\right|^{2 r} \mathrm{~d} x \leq \gamma^{2 r}\left(\int_{\Omega}\left|\nabla v_{\tau}^{i}\right|^{2} \mathrm{~d} x+1\right)^{r} \\
& \leq C(\Omega)^{2 r} \gamma^{2 r}\left(\int_{\Omega}\left|e\left(v_{\tau}^{i}\right)\right|^{2} \mathrm{~d} x+1\right)^{r}
\end{aligned}
$$

where $C(\Omega)$ is the Korn-Poincaré constant. In particular, all the above constants are independent of $\tau$ and $i$.

5.3. A priori estimates. Next, we obtain an apriori estimate for the piecewise constant interpolations $\left(z_{\tau}, v_{\tau}\right)$. that

Since $\left(z_{\tau}^{i-1}, v_{\tau}^{i-1}-\varphi_{\tau}^{i-1}+\varphi_{\tau}^{i}\right) \in L^{1}(\Omega) \times\left(\varphi_{\tau}^{i}+H_{0}^{1}\left(\Omega ; \mathbb{R}^{d}\right)\right)$, the minimality of $\left(z_{\tau}^{i}, v_{\tau}^{i}\right)$ implies

$$
\begin{aligned}
& \mathcal{W}\left(z_{\tau}^{i}, e\left(v_{\tau}^{i}\right)\right)+\mathcal{D}\left(z_{\tau}^{i-1}, z_{\tau}^{i}\right) \leq \mathcal{W}\left(z_{\tau}^{i-1}, e\left(v_{\tau}^{i-1}-\varphi_{\tau}^{i-1}+\varphi_{\tau}^{i}\right)\right) \\
= & \mathcal{W}\left(z_{\tau}^{i-1}, e\left(v_{\tau}^{i-1}\right)\right)+\mathcal{W}\left(z_{\tau}^{i-1}, e\left(v_{\tau}^{i-1}-\varphi_{\tau}^{i-1}+\varphi_{\tau}^{i}\right)\right)-\mathcal{W}\left(z_{\tau}^{i-1}, e\left(v_{\tau}^{i-1}\right)\right) .
\end{aligned}
$$


The last two terms of the right-hand side above may be controlled as follows

$$
\begin{aligned}
& \mathcal{W}\left(z_{\tau}^{i-1}, e\left(v_{\tau}^{i-1}-\varphi_{\tau}^{i-1}+\varphi_{\tau}^{i}\right)\right)-\mathcal{W}\left(z_{\tau}^{i-1}, e\left(v_{\tau}^{i-1}\right)\right) \\
= & \int_{t_{\tau}^{i-1}}^{t_{\tau}^{i}}\left[\int_{\Omega} \frac{\partial W}{\partial \varepsilon}\left(z_{\tau}^{i-1}, e\left(v_{\tau}^{i-1}-\varphi_{\tau}^{i-1}+\varphi(s)\right)\right): e(\dot{\varphi}(s)) \mathrm{d} x\right] \mathrm{d} s \\
= & \int_{t_{\tau}^{i-1}}^{t_{\tau}^{i}}\left[\int_{\Omega} \sigma_{\tau}(s): e(\dot{\varphi}(s)) \mathrm{d} x\right] \mathrm{d} s \\
& +\int_{t_{\tau}^{i-1}}^{t_{\tau}^{i}}\left[\int_{\Omega}\left(\frac{\partial W}{\partial \varepsilon}\left(z_{\tau}^{i-1}, e\left(v_{\tau}^{i-1}-\varphi_{\tau}^{i-1}+\varphi(s)\right)\right)-\frac{\partial W}{\partial \varepsilon}\left(z_{\tau}^{i-1}, e\left(v_{\tau}^{i-1}\right)\right)\right): e(\dot{\varphi}(s)) \mathrm{d} x\right] \mathrm{d} s
\end{aligned}
$$

Taking the sum in $(5.8)$ for $t \in[0, T], \tau(t):=\max \left\{t_{\tau}^{i}: t_{\tau}^{i} \leq t\right\}$, we have

$$
\begin{aligned}
& \mathcal{W}\left(z_{\tau}(t), e\left(v_{\tau}(t)\right)\right)+\operatorname{Diss}\left(z_{\tau} ; 0, t\right) \leq \mathcal{W}\left(z_{0}, v_{0}\right)+\int_{0}^{\tau(t)}\left[\int_{\Omega} \sigma_{\tau}(s): e(\dot{\varphi}(s)) \mathrm{d} x\right] \mathrm{d} s \\
& +\int_{0}^{\tau(t)}\left[\int_{\Omega}\left(\frac{\partial W}{\partial \varepsilon}\left(z_{\tau}(s), e\left(v_{\tau}(s)-\varphi_{\tau}(s)+\varphi(s)\right)\right)-\frac{\partial W}{\partial \varepsilon}\left(z_{\tau}(s), e\left(v_{\tau}(s)\right)\right)\right): e(\dot{\varphi}(s)) \mathrm{d} x\right] \mathrm{d} s .
\end{aligned}
$$

We observe that, thanks to $(W .3)$, we have

$$
\begin{aligned}
& \left|\int_{0}^{\tau(t)}\left[\int_{\Omega}\left(\frac{\partial W}{\partial \varepsilon}\left(z_{\tau}(s), e\left(v_{\tau}(s)-\varphi_{\tau}(s)+\varphi(s)\right)\right)-\frac{\partial W}{\partial \varepsilon}\left(z_{\tau}(s), e\left(v_{\tau}(s)\right)\right)\right): e(\dot{\varphi}(s)) \mathrm{d} x\right] \mathrm{d} s\right| \\
\leq & 2 C\left(\sup _{t \in[0, T]}\left\|e\left(v_{\tau}(t)\right)\right\|_{2}+\sup _{t \in[0, T]}\|e(\varphi(t))\|_{2}+1\right) \int_{0}^{T}\|e(\dot{\varphi}(s))\|_{2} \mathrm{~d} s .
\end{aligned}
$$

Now, $\int_{0}^{T}\|e(\dot{\varphi}(s))\|_{2} \mathrm{~d} s+\sup _{t \in[0, T]}\|e(\varphi(t))\|_{2}<\infty$, since $\varphi \in A C\left([0, T], H^{1}\left(\Omega ; \mathbb{R}^{d}\right)\right.$. Hence, also owing definition (5.6) and $(W .2)$, we get

$$
\begin{aligned}
c_{W} \sup _{t \in[0, T]}\left\|e\left(v_{\tau}(t)\right)\right\|_{2}^{2} & \leq \sup _{t \in[0, T]} \mathcal{W}\left(z_{\tau}(t), e\left(v_{\tau}(t)\right)\right)+\operatorname{Diss}\left(z_{\tau} ; 0, T\right) \\
& \leq \mathcal{W}\left(z_{0}, v_{0}\right)+C\left(\sup _{t \in[0, T]}\left\|e\left(v_{\tau}(t)\right)\right\|_{2}+1\right)
\end{aligned}
$$

for every $t \in[0, T]$ and a positive constant $C$. Therefore, we deduce that there exists a positive constant $K$, independent of the choice of the time step $\tau$, such that

$$
\sup _{t \in[0, T]}\left\|e\left(v_{\tau}(t)\right)\right\|_{2} \leq K
$$

In particular, we get

$$
\begin{aligned}
& \sup _{t \in[0, T]}\left\|\nabla v_{\tau}(t)\right\|_{2} \leq C(\Omega) K \\
& \sup _{t \in[0, T]}\left\|\sigma_{\tau}(t)\right\|_{2} \leq C_{W}(K+1) .
\end{aligned}
$$

Thanks to the improved regularity estimate (5.7), we get

$$
\sup _{t \in[0, T]}\left\|\nabla v_{\tau}(t)\right\|_{2 r} \leq C(\Omega) \gamma \sqrt{K^{2}+1} .
$$


5.4. Passage to the limit. Let us now consider a sequence of time steps $\left(\tau_{n}\right)_{n}$ converging to 0 , and the associated interpolations $\left(z_{\tau_{n}}, v_{\tau_{n}}\right)_{n}$. We want to define a family of measures $\nu \in A Y([0, T] ; \varphi)$. We will do this by passage to the limit in the sequence of approximate solutions $\left(z_{\tau_{n}}(t), v_{\tau_{n}}(t)\right)_{n}$. For technical reasons, which will appear patent in the proof, we need to proceed by defining $\nu_{t}$ on larger and larger time sets. In particular, we will first define $\nu_{t}$ for $t \in[0, T] \cap \mathbb{Q}$ and then in the rest of $[0, T]$.

Thanks to the uniform bound (5.10) and to the higher integrability estimate (5.12), and by using a diagonalization argument, we can find a not relabeled subsequence $\left(z_{\tau_{n}}, v_{\tau_{n}}\right)$ and $\tilde{\nu} \in Y^{2 r}\left(\Omega ; \mathbb{R} \times \mathbb{R}^{d \times d}\right)^{[0, T] \cap \mathbb{Q}}$, such that

$$
\boldsymbol{\delta}_{\left(z_{\tau_{n}}(t), \nabla v_{\tau_{n}}(t)\right)} \rightarrow \tilde{\nu}_{t} \quad 2 r \text {-weakly* }
$$

for every $t \in[0, T] \cap \mathbb{Q}$.

For every $t \in[0, T] \backslash \mathbb{Q}$, let us choose an increasing sequence of integers $n_{k}^{t}$ possibly depending on $t$, such that

$$
\limsup _{n}\left\langle\sigma_{\tau_{n}}(t), e(\dot{\varphi}(t))\right\rangle=\lim _{k}\left\langle\sigma_{\tau_{n_{k}^{t}}}(t), e(\dot{\varphi}(t))\right\rangle .
$$

Again, we are allowed to extract a further subsequence, still denoted by $\left(z_{\tau_{n_{k}^{t}}}, v_{\tau_{n_{k}^{t}}}\right)_{k}$ satisfying (5.13) and such that there exists $\tilde{\nu}_{t} \in Y^{2 r}\left(\Omega ; \mathbb{R} \times \mathbb{R}^{d \times d}\right)$ with

$$
\boldsymbol{\delta}_{\left(z_{n_{k}^{t}}(t), \nabla v_{\tau_{n} t}^{t}(t)\right)} \rightarrow \tilde{\nu}_{t} \quad 2 r \text {-weakly*, as } k \rightarrow \infty
$$

for every $t \in[0, T] \backslash \mathbb{Q}$. Note that, for every $t \in[0, T] \backslash \mathbb{Q}$,

$$
\begin{gathered}
\limsup _{n}\left\langle\sigma_{\tau_{n}}(t), e(\dot{\varphi}(t))\right\rangle=\lim _{k}\left\langle\sigma_{\tau_{n_{k}^{t}}}(t), e(\dot{\varphi}(t))\right\rangle \\
=\lim _{k} \int_{\Omega} \frac{\partial W}{\partial \varepsilon}\left(z_{\tau_{n_{k}^{t}}}(t), e\left(v_{\tau_{n_{k}^{t}}}(t)\right)\right): e(\dot{\varphi}(t)) \mathrm{d} x=\langle\sigma(t), e(\dot{\varphi}(t)\rangle,
\end{gathered}
$$

where $\sigma$ is defined by

$$
\sigma(t, x):=\int_{\mathbb{R} \times \mathbb{R}^{d \times d}} \frac{\partial W}{\partial \varepsilon}(\theta, \varepsilon) \mathrm{d} \tilde{\nu}_{t}^{x}(\theta, \varepsilon) .
$$

Moreover, for every $t \in[0, T] \cap \mathbb{Q}$ we have

$$
\limsup _{n}\left\langle\sigma_{\tau_{n}}(t), e(\dot{\varphi}(t))\right\rangle=\lim _{n}\left\langle\sigma_{\tau_{n}}(t), e(\dot{\varphi}(t))\right\rangle=\langle\sigma(t), e(\dot{\varphi}(t)\rangle .
$$

This implies that the map in 4.2 is measurable on $[0, T]$.

In this way, we have defined $\tilde{\nu}$ in $Y^{2 r}\left(\Omega ; \mathbb{R} \times \mathbb{R}^{d \times d}\right)^{[0, T]}$, satisfying by construction properties (3.23) and (3.24) in Definition 3.10. Therefore, by letting $\nu_{t}:=S \tilde{\nu}_{t}$ for every $t \in[0, T]$, we get that $\nu$ satisfies also condition (3.22), and hence $\nu \in A Y([0, T], \varphi)$.

In particular we have:

$$
\begin{array}{rl}
\boldsymbol{\delta}_{\left(z_{\tau_{n}}(t), e\left(v_{\tau_{n}}(t)\right)\right)} \rightarrow \nu_{t} & 2 r \text {-weakly*, for } t \in[0, T] \cap \mathbb{Q}, \\
\boldsymbol{\delta}_{\left(z_{\tau_{n_{k}^{t}}}(t), e\left(v_{\tau_{n_{k}^{t}}}(t)\right)\right)} \rightarrow \nu_{t} & 2 r \text {-weakly* for } t \in[0, T] \backslash \mathbb{Q} .
\end{array}
$$


Since $\left(z_{\tau_{n}}(0), v_{\tau_{n}}(0)\right)=\left(z_{0}, v_{0}\right)$ for every $n$, the initial condition $(E 0)$ is automatically satisfied.

5.5. Irreversibility. Let us consider $0 \leq s<t \leq T$ and fix $q \in[s, t] \cap \mathbb{Q}$.

Up to not relabeled subsequences, we have that there exist $\mu_{s q}, \mu_{q t} \in Y\left(\Omega ;[0,1]^{2}\right)$ with

$$
\begin{array}{cc}
\left.\boldsymbol{\delta}_{\left(z_{n_{k}^{s}}\right.}(s), z_{\tau_{n_{k}^{s}}}(q)\right) & \rightarrow \mu_{s q} \text { weakly* } \\
\boldsymbol{\delta}_{\left(z_{\tau_{k}^{t}}(q), z_{\tau_{n_{k}^{t}}}(t)\right)} \rightarrow \mu_{q t} & \text { weakly*. }
\end{array}
$$

Thanks to the construction of $\nu$, we have that $\mu_{s q}$ has projections $\pi_{1}\left(\nu_{s}\right)$ and $\pi_{1}\left(\nu_{q}\right)$, respectively, and $\mu_{q t}$ has projections $\pi_{1}\left(\nu_{q}\right)$ and $\pi_{1}\left(\nu_{t}\right)$, respectively.

Now, we have $z_{\tau_{n}}(t) \leq z_{\tau_{n}}(q) \leq z_{\tau_{n}}(s)$ almost everywhere in $\Omega$, for every $n$. This implies that $\boldsymbol{\delta}_{\left(z_{\tau_{n}}(s), z_{\tau_{n}}(q)\right)}\left(E \times\left\{\theta_{1}<\theta_{2}\right\}\right)=0$ and $\boldsymbol{\delta}_{\left(z_{\tau_{n}}(q), z_{\tau_{n}}(t)\right)}\left(E \times\left\{\theta_{1}<\theta_{2}\right\}\right)=0$ for $E \subseteq \Omega$ open, for every $n$.

Since $\boldsymbol{\delta}_{\left(z_{\tau_{k} s}^{s}(s), z_{\tau_{k} s}(q)\right)} \rightarrow \mu_{s q}$ weakly* as $n \rightarrow \infty$, and $E \times\left\{\theta_{1}<\theta_{2}\right\}$ is open, we have

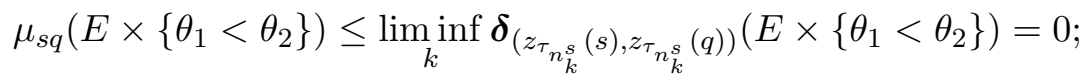

therefore $\mu_{s q}^{x}\left(\left\{\theta_{1}<\theta_{2}\right\}\right)=0$ for almost every $x \in \Omega$. The same holds for $\mu_{q t}: \mu_{q t}^{x}\left(\left\{\theta_{1}<\theta_{2}\right\}\right)=$ 0 for almost every $x \in \Omega$.

This implies, by Theorem 3.6. that $\pi_{1}\left(\nu_{s}\right) \succeq \pi_{1}\left(\nu_{q}\right)$ and $\pi_{1}\left(\nu_{q}\right) \succeq \pi_{1}\left(\nu_{t}\right)$. By transitivity, this implies $\pi_{1}\left(\nu_{s}\right) \succeq \pi_{1}\left(\nu_{t}\right)$, namely the irreversibility condition $(E 1)$.

5.6. Stability. Let $\tilde{z} \in L^{1}(\Omega)$ and $\tilde{u} \in H_{0}^{1}\left(\Omega ; \mathbb{R}^{d}\right)$. Let us observe that if $\tilde{z}>0$ on $\Omega^{\prime} \subseteq \Omega$ with $\left|\Omega^{\prime}\right|>0$, then $\mathbb{D}\left(\mu, \operatorname{Tr}_{\tilde{z}} \mu\right)=\infty$, for every $\mu \in Y(\Omega ;[0,1])$. Indeed, if $\mu \succeq \operatorname{Tr}_{\tilde{z}} \mu$, then for every $\alpha \in \mathbb{R}$ we would have $\mu^{x}(\alpha, \infty) \geq \operatorname{Tr}_{\tilde{z}(x)} \mu^{x}(\alpha, \infty)=\mu^{x}(\alpha-\tilde{z}(x), \infty)$. Therefore, for $x \in \Omega^{\prime}$, we would have $\mu^{x}(\alpha-\tilde{z}(x), \alpha]=0$, for every $\alpha \in \mathbb{R}$. This would imply $\mu^{x}([0,1])=0$, which is a contradiction with the fact that $\mu^{x}$ is a probability measure on $[0,1]$, for almost every $x$. In conclusion, if $\tilde{z}>0$ on a subset of $\Omega$ with positive measure, (E2) is automatically satisfied.

Hence, we reduce to the case $\tilde{z} \leq 0$ almost everywhere in $\Omega$. For every $n$ and every $i=1, \ldots, n$, the function $\left(z_{\tau_{n}}^{i}+\tilde{z}, v_{\tau_{n}}^{i}+\tilde{u}\right)$ is an admissible competitor for the minimum problem defining $\left(z_{\tau_{n}}^{i}, v_{\tau_{n}}^{i}\right)$. Therefore, we have

$$
\mathcal{W}\left(z_{\tau_{n}}^{i}, v_{\tau_{n}}^{i}\right)+\mathcal{D}\left(z_{\tau_{n}}^{i-1}, z_{\tau_{n}}^{i}\right) \leq \mathcal{W}\left(z_{\tau_{n}}^{i}+\tilde{z}, v_{\tau_{n}}^{i}+\tilde{u}\right)+\mathcal{D}\left(z_{\tau_{n}}^{i-1}, z_{\tau_{n}}^{i}+\tilde{z}\right)
$$

Since $\tilde{z} \leq 0$ almost everywhere in $\Omega$, we have that $z_{\tau_{n}}^{i}+\tilde{z} \leq z_{\tau_{n}}^{i} \leq z_{\tau_{n}}^{i-1}$. This implies that $\mathcal{D}\left(z_{\tau_{n}}^{i-1}, z_{\tau_{n}}^{i}-\tilde{z}\right)=\rho \int_{\Omega}\left(z_{\tau_{n}}^{i-1}-z_{\tau_{n}}^{i}+\tilde{z}\right) \mathrm{d} x$ and hence

$$
\mathcal{D}\left(z_{\tau_{n}}^{i-1}, z_{\tau_{n}}^{i}+\tilde{z}\right)-\mathcal{D}\left(z_{\tau_{n}}^{i-1}, z_{\tau_{n}}^{i}\right)=\mathcal{D}\left(z_{\tau_{n}}^{i}, z_{\tau_{n}}^{i}+\tilde{z}\right)=\mathcal{D}(0, \tilde{z})
$$

Hence, we get

$$
\mathcal{W}\left(z_{\tau_{n}}^{i}, v_{\tau_{n}}^{i}\right) \leq \mathcal{W}\left(z_{\tau_{n}}^{i}+\tilde{z}, v_{\tau_{n}}^{i}+\tilde{u}\right)+\mathcal{D}(0, \tilde{z})
$$

This means that, for every $t \in[0, T]$, we have

$$
\mathcal{W}\left(z_{\tau_{n}}(t), v_{\tau_{n}}(t)\right) \leq \mathcal{W}\left(z_{\tau_{n}}(t)+\tilde{z}, v_{\tau_{n}}(t)+\tilde{u}\right)+\mathcal{D}(0, \tilde{z})
$$


We observe that $\theta+\tilde{z}(x) \leq 1$ for a.e. $x \in \Omega$, for every $\theta \in[0,1](\tilde{z} \leq 0$ almost everywhere in $\Omega)$. Hence, thanks to $(W .2)$, we have

$$
|W(\theta+\tilde{z}(x), \varepsilon+e(\tilde{u}))| \leq C\left(|e(\tilde{u})(x)|^{2}+|\varepsilon|^{2}\right) .
$$

Therefore, by using the convergence (5.14) and Lemma 3.4 we get

$$
\begin{aligned}
\int_{\Omega} W\left(z_{\tau_{n_{k}^{t}}}(t), e\left(v_{\tau_{n_{k}^{t}}}(t)\right)\right) \mathrm{d} x & \longrightarrow \int_{\Omega \times \mathbb{R} \times \mathbb{R}_{\mathrm{sym}}^{d \times d}} W(\theta, \varepsilon) \mathrm{d} \nu_{t}(x, \theta, \varepsilon), \\
\int_{\Omega} W\left(z_{\tau_{n_{k}^{t}}}(t)+\tilde{z}(x), e\left(v_{\tau_{n_{k}^{t}}}(t)\right)+e(\tilde{u})(x)\right) \mathrm{d} x & \longrightarrow \int_{\Omega \times \mathbb{R} \times \mathbb{R}_{\mathrm{sym}}^{d \times d}} W(\theta+\tilde{z}(x), \varepsilon+e(\tilde{u})(x)) \mathrm{d} \nu_{t}(x, \theta, \varepsilon) \\
& =\left\langle W, \operatorname{Tr}_{(\tilde{z}, e(\tilde{u}))}\left(\nu_{t}\right)\right\rangle,
\end{aligned}
$$

for every $t \in(0, T]$, as $n \rightarrow \infty$. Therefore, we can deduce the translational stability (E2) passing to the limit in inequality (5.15). For $t=0$, relation $(E 2)$ comes immediately from the hypothesis on the initial datum 4.3 .

Now we want to prove the global stability for the internal variable $(E 3)$. Let us denote $\pi_{1}\left(\nu_{t}\right)$ by $\mu_{t}$, for every $t \in[0, T]$.

Let us start by proving (E3) for $\tilde{\mu} \in Y(\Omega ;[0,1])$. From the minimality of $\left(z_{\tau_{n}}^{i}, v_{\tau_{n}}^{i}\right)$, we get that for every $(\tilde{z}, \tilde{v}) \in L^{1}(\Omega) \times\left(\varphi_{\tau_{n}}^{i}+H_{0}^{1}\left(\Omega ; \mathbb{R}^{d}\right)\right)$,

$$
\mathcal{W}\left(z_{\tau_{n}}^{i}, v_{\tau_{n}}^{i}\right)+\mathcal{D}\left(z_{\tau_{n}}^{i-1}, z_{\tau_{n}}^{i}\right) \leq \mathcal{W}(\tilde{z}, \tilde{v})+\mathcal{D}\left(z_{\tau_{n}}^{i-1}, \tilde{z}\right)
$$

Hence, using the triangle inequality for $\mathcal{D}$, we get

$$
\mathcal{W}\left(z_{\tau_{n}}^{i}, v_{\tau_{n}}^{i}\right) \leq \mathcal{W}(\tilde{z}, \tilde{v})+\mathcal{D}\left(z_{\tau_{n}}^{i}, \tilde{z}\right)
$$

Therefore, we deduce that for every $n, t \in[0, T]$, and $(\tilde{z}, \tilde{v}) \in L^{1}(\Omega) \times\left(\varphi(t)+H_{0}^{1}\left(\Omega ; \mathbb{R}^{d}\right)\right)$, we have

$$
\begin{aligned}
\mathcal{W}\left(z_{\tau_{n}}(t), e\left(v_{\tau_{n}}(t)\right)\right) & \leq \mathcal{W}\left(\tilde{z}, e\left(\tilde{v}-\varphi(t)+\varphi_{\tau_{n}}(t)\right)\right)+\mathcal{D}\left(z_{\tau_{n}}(t), \tilde{z}\right) \\
& =\mathcal{W}(\tilde{z}, e(\tilde{v}))+\mathcal{D}\left(z_{\tau_{n}}(t), \tilde{z}\right)+R_{n}(t),
\end{aligned}
$$

where

$$
R_{n}(t):=\mathcal{W}\left(\tilde{z}, e\left(\tilde{v}-\varphi(t)+\varphi_{\tau_{n}}(t)\right)\right)-\mathcal{W}(\tilde{z}, e(\tilde{v})) .
$$

Arguing as in Subsection 5.3, it is not difficult to show that

$$
\left|R_{n}(t)\right| \leq 2 C\left(\sup _{t \in[0, T]}\|e(\varphi(t))\|_{2}+\|e(\tilde{v})\|_{2}+1\right) \int_{t-\tau_{n}}^{t}\|e(\dot{\varphi}(s))\|_{2} \mathrm{~d} s .
$$

Since $\dot{\varphi} \in L^{1}\left([0, T] ; H^{1}\left(\Omega ; \mathbb{R}^{d}\right)\right)$, we have that, for every $t \in[0, T]$

$$
R_{n}(t) \rightarrow 0, \quad \text { as } n \rightarrow \infty \text {. }
$$

Let us now fix $t \in[0, T]$ and a competitor $\tilde{\mu} \in Y(\Omega ;[0,1])$. If $\mu_{t} \nsucceq \tilde{\mu}$, we have $\mathbb{D}\left(\mu_{t}, \tilde{\mu}\right)=\infty$ and hence 4.1) holds true. So we can assume that $\mu_{t} \succeq \tilde{\mu}$. Thanks to Theorem 3.6, there exists a measure $\mu_{12, t}$ such that

$$
\begin{aligned}
& \pi_{1}\left(\mu_{12, t}\right)=\mu_{t}, \quad \pi_{2}\left(\mu_{12, t}\right)=\tilde{\mu}, \\
& \mu_{12, t}^{x}\left(\left\{\theta_{1}<\theta_{2}\right\}\right)=0 \quad \text { for a.e. } x \in \Omega .
\end{aligned}
$$


Let us consider the sequence $\left(z_{\tau_{n_{k}^{t}}}(t), v_{\tau_{n_{k}^{t}}}(t)\right)_{k}$ such that $\boldsymbol{\delta}_{\left(z_{\tau_{n_{k}^{t}}}(t), e\left(v_{\tau_{n_{k}^{t}}}(t)\right)\right)} \rightarrow \nu_{t} 2 r$-weakly* This implies, by Lemma 3.4, that

$$
\mathcal{W}\left(z_{\tau_{n_{k}^{t}}}(t), e\left(v_{\tau_{n_{k}^{t}}}(t)\right)\right)=\left\langle W, \boldsymbol{\delta}_{\left(z_{\tau_{n_{k}^{t}}}(t), e\left(v_{\tau_{n_{k}^{t}}}(t)\right)\right)}\right\rangle \rightarrow\left\langle W, \nu_{t}\right\rangle, \quad \text { as } k \rightarrow \infty .
$$

Moreover $\boldsymbol{\delta}_{z_{\tau_{k} t}(t)} \rightarrow \pi_{1}\left(\mu_{12, t}\right)$ weakly*, so we can apply Theorem 3.8 and Corollary 3.9 to construct a sequence $\left(\tilde{z}_{k}\right)_{k}$ in $L^{1}(\Omega ;[0,1])$ such that, as $k \rightarrow \infty$,

$$
\begin{aligned}
\tilde{z}_{k} & \leq z_{\tau_{n_{k}^{t}}}(t) \quad \text { a.e. in } \Omega, \\
\boldsymbol{\delta}_{\left(z_{n_{k}}(t), \tilde{z}_{k}\right)} & \rightarrow \mu_{12, t} \quad \text { weakly* } \\
\boldsymbol{\delta}_{\tilde{z}_{k}} & \rightarrow \tilde{\mu} \quad \text { weakly*. }
\end{aligned}
$$

We can apply Lemma 3.4 to obtain

$$
\begin{aligned}
\mathcal{W}\left(\tilde{z}_{k}, e(\tilde{v})\right) & =\int_{\Omega \times \mathbb{R}} W\left(\theta, e(\tilde{v}(x)) \mathrm{d} \boldsymbol{\delta}_{\tilde{z}_{k}}(x, \theta) \rightarrow\right. \\
& \rightarrow \int_{\Omega \times \mathbb{R}} W\left(\theta, e(\tilde{v}(x)) \mathrm{d} \tilde{\mu}(x, \theta)=\left\langle W,\left(\tilde{\mu}^{x} \otimes \boldsymbol{\delta}_{e(\tilde{v})(x)}\right)_{x \in \Omega}\right\rangle\right.
\end{aligned}
$$

as $k \rightarrow \infty$. As $\tilde{z}_{k} \leq z_{\tau_{n_{k}^{t}}}(t)$ almost everywhere in $\Omega$, we have that

$$
\begin{aligned}
\mathcal{D}\left(z_{\tau_{n_{k}^{t}}}(t), \tilde{z}_{k}\right) & =\int_{\Omega} d\left(z_{\tau_{n_{k}^{t}}}(x, t), \tilde{z}_{k}(x)\right) \mathrm{d} x=\int_{\Omega} \rho\left(z_{\tau_{n_{k}}}(x, t)-\tilde{z}_{k}(x)\right) \mathrm{d} x \\
& =\int_{\Omega \times \mathbb{R}^{2}} \rho\left(\theta_{1}-\theta_{2}\right) \mathrm{d} \boldsymbol{\delta}_{\left(z_{\tau_{n_{k}^{t}}}(t), \tilde{z}_{k}\right)} \\
& \rightarrow \int_{\Omega \times \mathbb{R}^{2}} \rho\left(\theta_{1}-\theta_{2}\right) \mathrm{d} \mu_{12, t}\left(x, \theta_{1}, \theta_{2}\right)=\rho\left[\int_{\Omega \times \mathbb{R}} \theta_{1} \mathrm{~d} \mu_{t}\left(x, \theta_{1}\right)-\int_{\Omega \times \mathbb{R}} \theta_{2} \mathrm{~d} \tilde{\mu}\left(x, \theta_{2}\right)\right] \\
& =\mathbb{D}\left(\mu_{t}, \tilde{\mu}\right)=\mathbb{D}\left(\pi_{1}\left(\nu_{t}\right), \tilde{\mu}\right),
\end{aligned}
$$

as $k \rightarrow \infty$.

Therefore, putting together inequality $(5.16)$ for $\tilde{z}=\tilde{z}_{k}$, and the convergence properties (5.17), 5.18), 5.19), and (5.20), we get 4.1).

Let us now consider a general $\hat{\mu} \in Y(\Omega ; \mathbb{R})$. If $\operatorname{supp}(\hat{\mu}) \nsubseteq \Omega \times(-\infty, 1]$, then $\mu_{t} \nsucceq \hat{\mu}$. Therefore, $\mathbb{D}\left(\mu_{t}, \hat{\mu}\right)=\infty$ and $(E 3)$ is proved. So let us assume that $\operatorname{supp}(\hat{\mu}) \subseteq \Omega \times(-\infty, 1]$. We define $\tilde{\mu} \in Y(\Omega ;[0,1])$, by setting:

$$
\int_{\Omega \times \mathbb{R}} f(x, \theta) \mathrm{d} \tilde{\mu}(x, \theta):=\int_{\Omega \times(0,1]} f(x, \theta) \mathrm{d} \hat{\mu}(x, \theta)+\int_{\Omega \times(-\infty, 0]} f(x, 0) \mathrm{d} \hat{\mu}(x, \theta),
$$

for every bounded Borel function $f: \Omega \times \mathbb{R} \rightarrow \mathbb{R}$. It is immediate to see that if $\mu_{t} \succeq \hat{\mu}$, then $\mu_{t} \succeq \tilde{\mu}$. Indeed, let $\alpha \in[0,1]$, then $\tilde{\mu}^{x}(\alpha, 1]=\hat{\mu}^{x}(\alpha, 1] \leq \mu_{t}^{x}(\alpha, 1]$, and, if $\alpha<0$, $\tilde{\mu}^{x}(\alpha, 1]=\tilde{\mu}^{x}[0,1]=1=\mu_{t}^{x}[0,1]=\mu_{t}^{x}(\alpha, 1]$, for a.e. $x \in \Omega$.

We claim that

$$
\left\langle W,\left(\tilde{\mu}^{x} \otimes \delta_{e(\tilde{v})(x)}\right)_{x \in \Omega}\right\rangle-\int_{\Omega \times \mathbb{R}} \theta \mathrm{d} \tilde{\mu}(x, \theta) \leq\left\langle W,\left(\hat{\mu}^{x} \otimes \delta_{e(\tilde{v})(x)}\right)_{x \in \Omega}\right\rangle-\int_{\Omega \times \mathbb{R}} \theta \mathrm{d} \hat{\mu}(x, \theta) .
$$


Indeed, we have

$$
\begin{aligned}
& \int_{\Omega \times \mathbb{R}} \theta \mathrm{d} \tilde{\mu}(x, \theta)=\int_{\Omega \times(0,1]} \theta \mathrm{d} \hat{\mu}(x, \theta)+\int_{\Omega \times(-\infty, 0]} 0 \mathrm{~d} \hat{\mu}(x, \theta) \\
\geq & \int_{\Omega \times(0,1]} \theta \mathrm{d} \hat{\mu}(x, \theta)+\int_{\Omega \times(-\infty, 0]} \theta \mathrm{d} \hat{\mu}(x, \theta)=\int_{\Omega \times \mathbb{R}} \theta \mathrm{d} \hat{\mu}(x, \theta) .
\end{aligned}
$$

On the other hand, thanks to $(W .5)$ we have

$$
\begin{aligned}
& \int_{\Omega \times \mathbb{R}} W(\theta, e(\tilde{v})(x)) \mathrm{d} \tilde{\mu}(x, \theta)=\int_{\Omega \times(0,1]} W(\theta, e(\tilde{v})(x)) \mathrm{d} \hat{\mu}(x, \theta)+\int_{\Omega \times(-\infty, 0]} W(0, e(\tilde{v})(x)) \mathrm{d} \hat{\mu}(x, \theta) \\
= & \int_{\Omega \times(0,1]} W(\theta, e(\tilde{v})(x)) \mathrm{d} \hat{\mu}(x, \theta)+\int_{\Omega \times(-\infty, 0]} W(\theta, e(\tilde{v})(x)) \mathrm{d} \hat{\mu}(x, \theta)=\int_{\Omega \times \mathbb{R}} W(\theta, e(\tilde{v})(x)) \mathrm{d} \hat{\mu}(x, \theta) .
\end{aligned}
$$

The claim is hence proved, and we have that

$$
\left\langle W,\left(\tilde{\mu}^{x} \otimes \delta_{e(\tilde{v})(x)}\right)_{x \in \Omega}\right\rangle+\mathbb{D}\left(\mu_{t}, \tilde{\mu}\right) \leq\left\langle W,\left(\hat{\mu}^{x} \otimes \delta_{e(\tilde{v})(x)}\right)_{x \in \Omega}+\mathbb{D}\left(\mu_{t}, \hat{\mu}\right)\right\rangle .
$$

Eventually we have checked that the global stability for the internal variable $(E 3)$ holds for $\tilde{\mu} \in Y(\Omega ; \mathbb{R})$ as well.

5.7. Upper energy estimate. First of all we observe that, thanks to the irreversibility property $(E 1)$ and Theorem 3.6 , we have, for every $t \in[0, T]$,

$$
\operatorname{Diss}(\nu ; 0, t)=\int_{\Omega \times \mathbb{R}} \rho\left(z_{0}(x)-\theta\right) \mathrm{d} \pi_{1}\left(\nu_{t}\right)(x, \theta) .
$$

Since $z_{\tau_{n}}(s) \geq z_{\tau_{n}}(t)$ almost everywhere in $\Omega$, whenever $s \leq t$, we have

$$
\operatorname{Diss}\left(z_{\tau_{n}} ; 0, t\right)=\int_{\Omega \times \mathbb{R}} \rho\left(z_{0}(x)-\theta\right) d \boldsymbol{\delta}_{z_{\tau_{n}}(t)}(x, \theta) .
$$

We have that $\boldsymbol{\delta}_{z_{\tau_{n_{k}^{t}}}(t)} \rightarrow \pi_{1}\left(\nu_{t}\right)$ weakly*, and hence we get $\operatorname{Diss}\left(z_{\tau_{n_{k}^{t}}} ; 0, t\right) \rightarrow \operatorname{Diss}(\nu ; 0, t)$ as $k \rightarrow \infty$. Let us fix $t \in[0, T]$. We have

$$
\left\langle W, \nu_{t}\right\rangle+\operatorname{Diss}(\nu ; 0, t) \leq \liminf _{k}\left[\mathcal{W}\left(z_{\tau_{n_{k}^{t}}}(t), e\left(v_{\tau_{n_{k}^{t}}}(t)\right)\right)+\operatorname{Diss}\left(z_{\tau_{n_{k}^{t}}} ; 0, t\right)\right] .
$$

By using estimate $(5.9)$, we deduce that

$$
\begin{aligned}
\left\langle W, \nu_{t}\right\rangle+\operatorname{Diss}(\nu ; 0, t) & \leq \liminf _{k}\left[\mathcal{W}\left(z_{0}, e\left(v_{0}\right)\right)+\int_{0}^{\tau_{n_{k}^{t}}(t)}\left\langle\sigma_{\tau_{n_{k}^{t}}}(s), e(\dot{\varphi}(s))\right\rangle \mathrm{d} s+\rho_{n_{k}^{t}}\right] \\
& \leq \mathcal{W}\left(z_{0}, e\left(v_{0}\right)\right)+\underset{n}{\lim \sup _{0}} \int_{0}^{\tau_{n}(t)}\left\langle\sigma_{n}(s), e(\dot{\varphi}(s))\right\rangle \mathrm{d} s+\underset{n}{\limsup \rho_{n}},
\end{aligned}
$$

where

$$
\rho_{n}:=\int_{0}^{\tau_{n}(t)}\left[\int_{\Omega}\left(\frac{\partial W}{\partial \varepsilon}\left(z_{\tau_{n}}(s), e\left(v_{\tau_{n}}(s)-\varphi_{\tau_{n}}(s)+\varphi(s)\right)\right)-\frac{\partial W}{\partial \varepsilon}\left(z_{\tau_{n}}(s), e\left(v_{\tau_{n}}(s)\right)\right)\right): e(\dot{\varphi}(s)) \mathrm{d} x\right] \mathrm{d} s .
$$


Since $\sup _{t, n}\left\|\sigma_{\tau_{n}}(t)\right\|_{2}$ is finite thanks to estimate (5.11), by Fatou Lemma we get

$$
\begin{aligned}
& \underset{n}{\limsup } \int_{0}^{\tau_{n}(t)}\left\langle\sigma_{n}(s), e(\dot{\varphi}(s))\right\rangle \mathrm{d} s \leq \int_{0}^{T} \limsup _{n} 1_{\left[0, \tau_{n}(t)\right]}\left\langle\sigma_{n}(s), e(\dot{\varphi}(s))\right\rangle \mathrm{d} s \\
& =\int_{0}^{t}\langle\sigma(s), e(\dot{\varphi}(s))\rangle \mathrm{d} s \text {. }
\end{aligned}
$$

Finally we apply the following lemma with $X=\Omega, H=\frac{\partial W}{\partial \varepsilon}, q=2, \Phi_{n}=\left(z_{\tau_{n}}(s), e\left(v_{\tau_{n}}(s)\right)\right)$, $\Psi_{n}:=\left(0, e\left(\varphi_{\tau_{n}}(s)-\varphi(s)\right)\right)$, and $\Phi=e(\dot{\varphi}(s))$.

Lemma 5.3. [10, Lemma 4.9] Let $(X, \mathcal{A}, \mu)$ be a finite measure space, let $q>1$, let $m, n \geq 1$, and let $H: X \times \mathbb{R}^{N} \rightarrow \mathbb{R}^{m}$ be a Carathéodory function. Assume that there exist a constant $a \geq 0$ and a nonnegative function $b \in L^{q^{\prime}}(X)$, with $q^{\prime}=q /(q-1)$, such that

$$
|H(x, \xi)| \leq a|\xi|^{q-1}+b(x)
$$

for every $(x, \xi) \in X \times \mathbb{R}^{N}$. Let $\Phi_{n}$ and $\Psi_{n}$ be two sequences in $L^{q}\left(X ; \mathbb{R}^{N}\right)$. Assume that $\Phi_{n}$ is bounded in $L^{q}\left(X ; \mathbb{R}^{N}\right)$ and $\Psi_{n}$ converges to 0 strongly in $L^{q}\left(X ; \mathbb{R}^{N}\right)$. Then

$$
\int_{X}\left[H\left(x, \Phi_{n}(x)+\Psi_{n}(x)\right)-H\left(x, \Phi_{n}(x)\right)\right] \Phi(x) \mathrm{d} \mu(x) \rightarrow 0
$$

for every $\Phi \in L^{q}\left(X ; \mathbb{R}^{m}\right)$.

We obtain

$$
\int_{\Omega}\left(\frac{\partial W}{\partial \varepsilon}\left(z_{\tau_{n}}(s), e\left(v_{\tau_{n}}(s)-\varphi_{\tau_{n}}(s)+\varphi(s)\right)\right)-\frac{\partial W}{\partial \varepsilon}\left(z_{\tau_{n}}(s), e\left(v_{\tau_{n}}(s)\right)\right)\right): e(\dot{\varphi}(s)) \mathrm{d} x \rightarrow 0,
$$

as $n \rightarrow \infty$, for a.e. $s \in[0, T]$. Moreover, we have

$$
\begin{aligned}
& \left|\int_{\Omega}\left(\frac{\partial W}{\partial \varepsilon}\left(z_{\tau_{n}}(s), e\left(v_{\tau_{n}}(s)-\varphi_{\tau_{n}}(s)+\varphi(s)\right)\right)-\frac{\partial W}{\partial \varepsilon}\left(z_{\tau_{n}}(s), e\left(v_{\tau_{n}}(s)\right)\right)\right): e(\dot{\varphi}(s)) \mathrm{d} x\right| \\
\leq & \tilde{c}\left(\sup _{n, t}\left\|e\left(v_{\tau_{n}}(t)\right)\right\|_{2}+\sup _{t} \int_{t-\tau_{n}}^{t}\|e(\dot{\varphi}(s))\|_{2} \mathrm{~d} s+1\right)\|e(\dot{\varphi}(s))\|_{2} \leq C\|e(\dot{\varphi}(s))\|_{2} \in L^{1}([0, T]),
\end{aligned}
$$

for a.e. $s \in[0, T]$. Therefore, by Dominated Convergence we get $\lim _{n} \rho_{n}=0$ and we can deduce that

$$
\left\langle W, \nu_{t}\right\rangle+\operatorname{Diss}(\nu ; 0, t) \leq \mathcal{W}\left(z_{0}, e\left(v_{0}\right)\right)+\int_{0}^{t}\langle\sigma(s), e(\dot{\varphi}(s))\rangle \mathrm{d} s
$$

5.8. Lower energy estimate. To prove the lower energy estimate, we proceed in the same way as in [12, Subsection 7.6]. We recall the main passages for the Reader's convenience. Let us denote $\pi_{1}\left(\nu_{t}\right)$ by $\mu_{t}$ for every $t \in[0, T]$. Let $s<t$, with $s \in[0, T] \cap \mathbb{Q}$ and $t \in[0, T]$. Thanks to the minimality property satisfied by $\left(z_{\tau_{n}}, v_{\tau_{n}}\right)$, the fact that $z_{\tau_{n}}(s) \geq z_{\tau_{n}}(t)$ almost everywhere in $\Omega$, and the triangle inequality for $\mathcal{D}$, we get

$$
\mathcal{W}\left(z_{\tau_{n}}(s), e\left(v_{\tau_{n}}(s)\right)\right) \leq \mathcal{W}\left(z_{\tau_{n}}(t), e\left(v_{\tau_{n}}(t)-\varphi(t)+\varphi(s)\right)\right)+\mathcal{D}\left(z_{\tau_{n}}(s), z_{\tau_{n}}(t)\right)+R_{n}(s, t),
$$

where now

$$
R_{n}(s, t):=\mathcal{W}\left(z_{\tau_{n}}(t), e\left(v_{\tau_{n}}(t)+\varphi_{\tau_{n}}(s)-\varphi_{\tau_{n}}(t)\right)\right)-\mathcal{W}\left(z_{\tau_{n}}(t), e\left(v_{\tau_{n}}(t)-\varphi(t)+\varphi(s)\right)\right) .
$$


As in Subsection 5.3, it is easy to see that $R_{n}(s, t) \rightarrow 0$ as $n \rightarrow \infty$.

Since $s \in[0, T] \cap \mathbb{Q}$, we have

$$
\begin{aligned}
& \boldsymbol{\delta}_{\left(z_{\tau_{n}}(s), e\left(v_{\tau_{n}}(s)\right)\right)} \rightarrow \nu_{s} \quad 2 r \text {-weakly* as } n \rightarrow \infty, \\
& \boldsymbol{\delta}_{\left(z_{n_{k}^{t}}(t), e\left(v_{\tau_{n_{k}^{t}}}(t)\right)\right)} \rightarrow \nu_{t} \quad 2 r \text {-weakly* as } k \rightarrow \infty,
\end{aligned}
$$

where $n_{k}^{t}$ is the subsequence chosen in Subsection 5.4 , if $t \notin[0, T] \cap \mathbb{Q}$.

Hence, passing to the limit in inequality $(5.22)$ we get

$$
\left\langle W, \nu_{s}\right\rangle \leq\left\langle W, \nu_{t}\right\rangle+\mathbb{D}\left(\mu_{s}, \mu_{t}\right)-\int_{s}^{t}\langle\sigma(\tau), e(\dot{\varphi}(\tau))\rangle \mathrm{d} \tau+R(s, t)
$$

where

$$
R(s, t):=\int_{s}^{t}\left\{\int_{\Omega \times \mathbb{R} \times \mathbb{R}^{d \times d}}\left[-\frac{\partial W}{\partial \varepsilon}(\theta, \varepsilon+e(\varphi(\tau)-\varphi(t)))+\frac{\partial W}{\partial \varepsilon}(\theta, \varepsilon)\right]: e(\dot{\varphi}(\tau)) \mathrm{d} \nu_{t}(x, \theta, \varepsilon)\right\} \mathrm{d} \tau .
$$

By changing the choice of the subsequence in (5.23), we obtain inequality (5.24) for $s \in[0, T]$ and $t \in[0, T] \cap \mathbb{Q}$.

Now we use a measure theoretic result (see [10], or [9, Lemma 4.12] for a detailed proof), which allows us to approximate a Lebesgue integral by Riemann sums. For the Reader's convenience we recall the statement of this result in the formulation of [12].

Lemma 5.4. Let $X$ be a Banach space, and let $F:[0, t] \rightarrow X$ be a Bochner integrable function. Then, there exists a sequence of partitions $\mathcal{S}_{j}:=\left\{s_{j}^{i}, 0 \leq i \leq n_{j}\right\}, j \in \mathbb{N}$ of the interval $[0, t]$, with

$$
\begin{gathered}
0=s_{j}^{0}<\cdots<s_{j}^{n_{j-1}}<s_{j}^{n_{j}}=t, \\
s_{j}^{1} \leq 1 / j, \quad t-s_{j}^{n_{j}-1} \leq 1 / j, \\
s_{j}^{i}-s_{j}^{i-1}=1 / j \quad \text { for } i=2, \ldots, n_{j}-1,
\end{gathered}
$$

such that

$$
\lim _{j} \sum_{i=1}^{n_{j}} \int_{s_{j}^{i-1}}^{s_{j}^{i}}\left\|F\left(s_{j}^{i}\right)-F(\tau)\right\| \mathrm{d} \tau=0 .
$$

We apply this Lemma to the functional defined by

$$
F \quad: \quad[0, t] \ni \tau \mapsto(e(\dot{\varphi}(\tau)),\langle\sigma(\tau), e(\dot{\varphi}(\tau))\rangle) \in L^{2}\left(\Omega ; \mathbb{R}^{d}\right) \times \mathbb{R}
$$

in order to find a sequence of partitions $\mathcal{S}_{j}$ of $[0, t]$ satisfying requirements $(5.25)$ and $(5.26)$, and such that

$$
\begin{aligned}
\lim _{j} \sum_{i=1}^{n_{j}} \int_{s_{j}^{i-1}}^{s_{j}^{i}}\left\|e\left(\dot{\varphi}\left(s_{j}^{i}\right)-\dot{\varphi}(\tau)\right)\right\|_{2} \mathrm{~d} \tau & =0, \\
\lim _{j} \sum_{i=1}^{n_{j}} \int_{s_{j}^{i-1}}^{s_{j}^{i}}\left|\left\langle\sigma\left(s_{j}^{i}\right), e\left(\dot{\varphi}\left(s_{j}^{i}\right)\right)\right\rangle-\langle\sigma(\tau), e(\dot{\varphi}(\tau))\rangle\right| \mathrm{d} \tau & =0 .
\end{aligned}
$$


Whenever both $s_{j}^{i-1}$ and $s_{j}^{i}$ belong to $[0, T] \backslash \mathbb{Q}$, we consider $t_{j}^{i-1} \in\left(s_{j}^{i-1}, s_{j}^{i-1}+1 / j^{2}\right) \cap \mathbb{Q}$, so that the estimate 5.24 holds true for $s_{j}^{i-1}, t_{j}^{i-1}$ and $t_{j}^{i-1}, s_{j}^{i}$. Hence we get

$$
\begin{aligned}
\left\langle W, \nu_{s_{j}^{i-1}}\right\rangle \leq & \left\langle W, \nu_{s_{j}^{i}}\right\rangle+\mathbb{D}\left(\mu_{s_{j}^{i-1}}, \mu_{t_{j}^{i-1}}\right)+\mathbb{D}\left(\mu_{t_{j}^{i-1}}, \mu_{s_{j}^{i}}\right) \\
& -\int_{s_{j}^{i-1}}^{s_{j}^{i}}\left\langle\sigma\left(s_{j}^{i}\right), e(\dot{\varphi}(\tau))\right\rangle \mathrm{d} \tau-\int_{s_{j}^{i-1}}^{t_{j}^{i-1}}\left\langle\left(\sigma\left(t_{j}^{i-1}\right)-\sigma\left(s_{j}^{i}\right)\right), e(\dot{\varphi}(\tau))\right\rangle \mathrm{d} \tau \\
& +R\left(s_{j}^{i-1}, t_{j}^{i-1}\right)+R\left(t_{j}^{i-1}, s_{j}^{i}\right) .
\end{aligned}
$$

Summing up with respect to $i$ and using $(E 0)$, we get

$$
\begin{aligned}
\mathcal{W}\left(z_{0}, e\left(v_{0}\right)\right)-\left\langle W, \nu_{t}\right\rangle-\operatorname{Diss}(\nu ; 0, t) \leq & -\sum_{i=1}^{i_{j}} \int_{s_{j}^{i-1}}^{s_{j}^{i}}\left\langle\sigma\left(s_{j}^{i}\right), e(\dot{\varphi}(\tau))\right\rangle \mathrm{d} \tau \\
& -\sum_{i=1}^{i_{j}} \int_{s_{j}^{i-1}}^{t_{j}^{i-1}}\left\langle\left(\sigma\left(t_{j}^{i-1}\right)-\sigma\left(s_{j}^{i}\right)\right), e(\dot{\varphi}(\tau))\right\rangle \mathrm{d} \tau \\
& +\sum_{i=1}^{i_{j}}\left[R\left(s_{j}^{i-1}, t_{j}^{i-1}\right)+R\left(t_{j}^{i-1}, s_{j}^{i}\right)\right] .
\end{aligned}
$$

By arguing as in [11, Lemma 7.5], we deduce that

$$
\sum_{i=1}^{i_{j}}\left[R\left(s_{j}^{i-1}, t_{j}^{i-1}\right)+R\left(t_{j}^{i-1}, s_{j}^{i}\right)\right] \rightarrow 0 \quad \text { as } j \rightarrow \infty .
$$

We now use Hölder inequality and the fact that $\sup _{t}\|\sigma(t)\|_{2}$ is bounded by estimate (5.11), in order to deduce that

$$
\left|\sum_{i=1}^{i_{j}} \int_{s_{j}^{i-1}}^{t_{j}^{i-1}}\left\langle\left(\sigma\left(t_{j}^{i-1}\right)-\sigma\left(s_{j}^{i}\right)\right), e(\dot{\varphi}(\tau))\right\rangle \mathrm{d} \tau\right| \rightarrow 0 \quad \text { as } j \rightarrow \infty .
$$

We have

$$
\begin{aligned}
& \left|\sum_{i=1}^{i_{j}} \int_{s_{j}^{i-1}}^{s_{j}^{i}}\left\langle\sigma\left(s_{j}^{i}\right), e(\dot{\varphi}(\tau))\right\rangle \mathrm{d} \tau-\int_{0}^{t}\langle\sigma(\tau), e(\dot{\varphi}(\tau))\rangle \mathrm{d} \tau\right| \\
\leq & \left|\sum_{i=1}^{i_{j}} \int_{s_{j}^{i-1}}^{s_{j}^{i}}\left\langle\sigma\left(s_{j}^{i}\right), e(\dot{\varphi}(\tau))\right\rangle \mathrm{d} \tau-\sum_{i=1}^{i_{j}} \int_{s_{j}^{i-1}}^{s_{j}^{i}}\left\langle\sigma\left(s_{j}^{i}\right), e\left(\dot{\varphi}\left(s_{j}^{i}\right)\right)\right\rangle \mathrm{d} \tau\right| \\
+ & \left|\sum_{i=1}^{i_{j}} \int_{s_{j}^{i-1}}^{s_{j}^{i}}\left\langle\sigma\left(s_{j}^{i}\right), e\left(\dot{\varphi}\left(s_{j}^{i}\right)\right)\right\rangle \mathrm{d} \tau-\int_{0}^{t}\langle\sigma(\tau), e(\dot{\varphi}(\tau))\rangle \mathrm{d} \tau\right| .
\end{aligned}
$$

Using properties (5.27) and (5.28) it is now possible to show that both the two last lines of 5.29) converge to 0 as $j \rightarrow \infty$, and hence we get

$$
\mathcal{W}\left(z_{0}, e\left(v_{0}\right)\right)+\int_{0}^{t}\langle\sigma(\tau), e(\dot{\varphi}(\tau))\rangle \mathrm{d} \tau \leq\left\langle W, \nu_{t}\right\rangle+\operatorname{Diss}(\nu ; 0, t)
$$

which, together with inequality (5.21), gives $(E 4)$. 
Remark 5.5. (Properties of the barycentre of the evolution) Let $W$ be a convex function, $\varphi \in A C\left([0, T] ; W^{1, p}\left(\Omega ; \mathbb{R}^{d}\right)\right), p>2, z_{0} \in L^{1}(\Omega ;[0,1]), v_{0} \in \varphi(0)+H_{0}^{1}\left(\Omega ; \mathbb{R}^{d}\right)$, and $\left(\nu_{t}\right)_{t \in[0, T]}$ be a damage quasi-static evolution. Let $\left(z_{b}(t), e\left(v_{b}(t)\right)\right.$ be the barycentre of $\nu_{t}$, for every $t$. A natural question is whether $\left(z_{b}(t), e\left(v_{b}(t)\right)\right.$ can be seen as a quasi-static evolution too. Let us focus on the stability condition. Thanks to Jensen's inequality, the global stability for the internal variable $(E 3)$, satisfied by $\nu_{t}$, gives

$$
\mathcal{W}\left(z_{b}(t), e\left(v_{b}(t)\right)\right) \leq\left\langle W, \nu_{t}\right\rangle \leq\left\langle W,\left(\tilde{\mu}^{x} \otimes \delta_{e(\tilde{v}(x))}\right)\right\rangle+\rho\left[\int_{\Omega \times \mathbb{R}} \theta \mathrm{d} \mu_{t}(x, \theta)-\int_{\Omega \times \mathbb{R}} \theta \mathrm{d} \tilde{\mu}(x, \theta)\right],
$$

for every $\tilde{\mu}$ with $\mu_{t} \succeq \tilde{\mu}$ and every $\tilde{v} \in \varphi(t)+H_{0}^{1}\left(\Omega ; \mathbb{R}^{d}\right)$. In particular, let us consider $\tilde{\mu}:=\boldsymbol{\delta}_{\tilde{z}}$, for $\tilde{z} \in L^{1}(\Omega)$; we get

$$
\mathcal{W}\left(z_{b}(t), e\left(v_{b}(t)\right)\right) \leq \mathcal{W}(\tilde{z}, e(\tilde{v}))+\int_{\Omega} \rho\left(z_{b}(t)-\tilde{z}\right) \mathrm{d} x
$$

whenever $\mu_{t} \succeq \boldsymbol{\delta}_{\tilde{z}}$. Since $\mu_{t} \succeq \boldsymbol{\delta}_{\tilde{z}}$ implies $z_{b}(t) \geq \tilde{z}$ almost everywhere in $\Omega$ (see Remark 3.7), we get

$$
\mathcal{W}\left(z_{b}(t), e\left(v_{b}(t)\right)\right) \leq \mathcal{W}(\tilde{z}, e(\tilde{v}))+\mathcal{D}\left(z_{b}(t), \tilde{z}\right) .
$$

Unfortunately, as observed in Remark 3.7, it may happen that $\tilde{z} \leq z_{b}(t)$ almost everywhere in $\Omega$, but $\mu_{t} \nsucceq \boldsymbol{\delta}_{\tilde{z}}$. Therefore, the minimality condition $(5.30)$ is true, again, only for restricted class of competitors $\tilde{z}$ (specifically, for those with $\mu_{t} \succeq \boldsymbol{\delta}_{\tilde{z}}$ ), and it is not the desired complete stability property.

\section{ACKNOWLEDGMENTS}

The authors would like to warmly thank Gianni Dal Maso for a crucial lead toward the proof of Theorem 3.8 and Gilles Francfort for some interesting discussions. This work is partially financed by the FP7-IDEAS-ERC-StG Grant 200497 BioSMA: Mathematics for Shape Memory Technologies in Biomechanics and by the PRIN08 Grant Optimal transport theory, geometric and functional inequalities and applications.

\section{APPENDIX}

In this appendix, we prove a result which has been used in the proof of Theorem 3.6 in order to construct a discrete version of a Young measure coupling two other given measures.

Theorem 5.6. (Matrix reconstruction) Fixed $n \in \mathbb{N}$, let $\left(A_{i}\right)_{i=1}^{n},\left(B_{j}\right)_{j=1}^{n}$ be two vectors in $[0,1]^{n}$ satisfying the following conditions:

$$
\begin{aligned}
\sum_{i=1}^{k} A_{i} & \leq \sum_{j=1}^{k} B_{j} \quad \text { for every } k \leq n, \\
\sum_{i=1}^{n} A_{i} & =\sum_{j=1}^{n} B_{j} .
\end{aligned}
$$


Then there exist a matrix $\left(C_{i j}\right)_{i, j=1}^{n}$ with entries in $[0,1]$ such that

$$
\begin{aligned}
\sum_{i=1}^{n} C_{i j} & =B_{j}, \\
\sum_{j=1}^{n} C_{i j} & =A_{i}, \\
C_{i j} & =0 \text { if } i<j .
\end{aligned}
$$

The following lemma will be used to prove Theorem 5.6, by induction.

Lemma 5.7. (Iteration) Given two vectors $\left(A_{i}\right)_{i=1}^{n}$ and $\left(B_{j}\right)_{j=1}^{n}$ in $[0,1]^{n}$ satisfying assumptions 5.31) and (5.32), there exists a vector $\left(C_{i 1}\right)_{i=1}^{n}$ in $[0,1]^{n}$ such that

$$
\begin{aligned}
& C_{11}=A_{1}, \\
& C_{i 1} \leq A_{i} \quad \text { for every } i \\
& \sum_{i=1}^{n} C_{i 1}=B_{1}, \\
& \sum_{i=2}^{k}\left(A_{i}-C_{i 1}\right) \leq \sum_{j=2}^{k} B_{j}, \quad \text { for every } 2 \leq k \leq n, \\
& \sum_{i=2}^{n}\left(A_{i}-C_{i 1}\right)=\sum_{j=2}^{n} B_{j} .
\end{aligned}
$$

Proof. According to (5.36), let us recursively define

$$
C_{11}:=A_{1}, \quad C_{i 1}:=A_{i}-\left[A_{i}-\left(B_{1}-\sum_{k=1}^{i-1} C_{k 1}\right)\right]^{+}, \text {for } i>1 .
$$

We observe that $B_{1}-C_{11}=B_{1}-A_{1} \geq 0$ by assumption (5.31), and that for $i>2$

$$
\begin{aligned}
B_{1}-\sum_{k=1}^{i-1} C_{k 1} & =B_{1}-\sum_{k=1}^{i-2} C_{k 1}-C_{i-1,1} \\
& =B_{1}-\sum_{k=1}^{i-2} C_{k 1}-\left\{A_{i-1}-\left[A_{i-1}-\left(B_{1}-\sum_{k=1}^{i-2} C_{k 1}\right)\right]^{+}\right\} \\
& =-\left[A_{i-1}-\left(B_{1}-\sum_{k=1}^{i-2} C_{k 1}\right)\right]+\left[A_{i-1}-\left(B_{1}-\sum_{k=1}^{i-2} C_{k 1}\right)\right]^{+} \\
& =0 \vee\left\{-\left[A_{i-1}-\left(B_{1}-\sum_{k=1}^{i-2} C_{k 1}\right)\right]\right\} \geq 0
\end{aligned}
$$

In particular, we have $A_{i}-\left(B_{1}-\sum_{k=1}^{i-2} C_{k 1}\right) \leq A_{i}$ and $0 \leq C_{i 1}=A_{i}-\left[A_{i}-\left(B_{1}-\sum_{k=1}^{i-2} C_{k 1}\right)\right]^{+} \leq$ $A_{i} \leq 1$. Hence, $C_{i 1} \in[0,1]$ for every $i$, and condition (5.37) holds true. 
Now, we show that there exists $i$ such that $C_{i 1}=B_{1}-\sum_{k=1}^{i-1} C_{k 1}$. By contradiction, let us suppose that for every $i=1, \ldots, n$ we have $C_{i 1}=A_{i}$ and hence $A_{i}<B_{1}-\sum_{k=1}^{i-1} C_{k 1}$. In particular, thanks to assumption 5.32 , we have

$$
A_{n}<B_{1}-\sum_{k=1}^{n-1} C_{k 1}=B_{1}-\sum_{k=1}^{n-1} A_{k}=B_{1}-\sum_{k=1}^{n} B_{k}+A_{n}=-\sum_{k=2}^{n} B_{k}+A_{n},
$$

which is a contradiction since $B_{j} \geq 0$ for every $j$. Hence, there exists $\bar{i}$ such that $A_{\bar{i} 1}=$ $B_{1}-\sum_{k=1}^{\bar{i}-1} C_{k 1}$. This implies that $C_{i 1}=0$ for every $i>\bar{i}$ and that $\sum_{i=1}^{n} C_{i 1}=\sum_{i=1}^{\bar{i}} C_{i 1}=$ $\sum_{i=1}^{\bar{i}-1} C_{i 1}+B_{1}-\sum_{i=1}^{\bar{i}-1} C_{i 1}=B_{1}$, so condition 5.38 is satisfied.

Using $C_{11}=A_{1}$ and (5.38), we obtain condition (5.40). Indeed, we have

$$
\sum_{i=2}^{n}\left(A_{i}-C_{i 1}\right)=\sum_{i=1}^{n} A_{i}-\sum_{i=1}^{n} C_{i 1}=\sum_{i=1}^{n} A_{i}-B_{1}=\sum_{j=1}^{n} B_{j}-B_{1}=\sum_{j=2}^{n} B_{j} .
$$

It remains only to show inequality $(5.39)$. We prove it by induction on $k$. For $k=2$, we have $A_{2}-C_{21}=\left[A_{2}-\left(B_{1}-A_{1}\right)\right]^{+}=\left[A_{1}+A_{2}-B_{1}\right]^{+}=0 \vee\left[A_{1}+A_{2}-B_{1}\right] \leq 0 \vee B_{2}=B_{2}$, thanks to assumption (5.31). Let us now assume that inequality (5.39) holds for $k-1$. Thanks to condition (5.37) and assumtpion (5.31), we have

$$
\begin{aligned}
& \sum_{i=2}^{k}\left(A_{i}-C_{i 1}\right)=\sum_{i=2}^{k-1}\left(A_{i}-C_{i 1}\right)+A_{k}-C_{k 1} \\
= & \sum_{i=2}^{k-1}\left(A_{i}-C_{i 1}\right)+\left[A_{k}-\left(B_{1}-\sum_{i=1}^{k-1} C_{i 1}\right)\right]^{+} \\
= & \sum_{i=2}^{k-1}\left(A_{i}-C_{i 1}\right)+\left[0 \vee\left(A_{k}-B_{1}+\sum_{i=1}^{k-1} C_{i 1}\right)\right] \\
\leq & \sum_{i=2}^{k-1}\left(A_{i}-C_{i 1}\right) \vee\left(\sum_{i=1}^{k} A_{i}-B_{1}\right) \leq \sum_{i=2}^{k-1}\left(A_{i}-C_{i 1}\right) \vee \sum_{j=2}^{k} B_{j} ;
\end{aligned}
$$

the inductive hypothesis implies that $\sum_{i=2}^{k-1}\left(A_{i}-C_{i 1}\right) \leq \sum_{j=2}^{k-1} B_{j} \leq \sum_{j=2}^{k} B_{j}$, and hence we can conclude that (5.39) holds true for every $k \geq 2$.

We are now able to prove Theorem 5.6 .

Proof of Theorem 5.6. For $j=1$ we define $C_{i 1}$ as in Lemma 5.7. For $2 \leq j \leq n$, we repeat the construction of Lemma 5.7, with $\left(A_{i}\right)_{i=1}^{n},\left(B_{j}\right)_{j=1}^{n}$ substituted by the vectors $\left(A_{i}-\sum_{k=1}^{j-1} C_{i k}\right)_{i=j}^{n}$ and $\left(B_{k}\right)_{k=j}^{n}$. Thanks to properties $(5.39)$ and $(5.40)$ we can prove by induction that the vectors $\left(A_{i}-\sum_{k=1}^{j-1} C_{i k}\right)_{i=j}^{n}$ and $\left(B_{k}\right)_{k=j}^{n}$ satisfy the assumption of the lemma. For $i<j$, we define $C_{i j}:=0$, so that condition 5.35 is satisfied. Due to identity (5.38), condition (5.33) holds true for every $j$. Thanks to this construction, we have $C_{i i}=A_{i}-\sum_{k=1}^{i-1} C_{i k}$ for every $i$. In 
particular, $\sum_{j=1}^{n} C_{i j}=\sum_{j=1}^{i} C_{i j}=C_{i i}+\sum_{j=1}^{i-1} C_{i j}=A_{i}$, for every $i$, and therefore property (5.34) is fulfilled.

\section{REFERENCES}

[1] H. Attouch, G. Buttazzo, G. Michaille. Variational analysis in Sobolev and BV spaces. Applications to PDEs and optimization. MPS/SIAM Series on Optimization, 6. Society for Industrial and Applied Mathematics (SIAM), Philadelphia, PA; Mathematical Programming Society (MPS), Philadelphia, PA, 2006.

[2] J.-F. Babadjian. A quasi-static evolution model for the interaction between fracture and damage. Arch. Rational Mech. Anal. To appear.

[3] G. Bouchitté, A. Mielke, and T. Roubíček. A complete-damage problem at small strains. ZAMP Z. Angew. Math. Phys. 60 (2009), 205-236.

[4] F. Cagnetti, R. Toader. Quasistatic crack evolution for a cohesive zone model with different response to loading and unloading: a Young measures approach. ESAIM Control Optim. Calc. Var. To appear.

[5] E.A. De Souza Neto, D. Peric, D.R.J. Owen. A phenomenological three-dimensional rate-independent continuum damage model for highly filled polymers: Formulation and computational aspects. J. Mech. Phys. Solids 42 (1994), 1533-1550.

[6] G. Dal Maso, A. De Simone, M.G. Mora, M. Morini. A vanishing viscosity approach to quasi-static evolution in plasticity with softening. Arch. Rational Mech. Anal. 189 (2008), 469-544.

[7] G. Dal Maso, A. De Simone, M.G. Mora and M. Morini. Time-dependent systems of generalized Young measures. Netw. Heterog. Media 2 (2007), 1-36.

[8] G. Dal Maso, A. De Simone, M.G. Mora and M. Morini. Globally stable quasi-static evolution in plasticity with softening. Netw. Heterog. Media 3 (2008), 567-614.

[9] G. Dal Maso, G. Francfort, R. Toader. Quasi-static crack growth in finite elasticity. Preprint SISSA, Trieste, 2004 (http://www.sissa.it/fa/).

[10] G. Dal Maso, G. Francfort and R. Toader. Quasistatic crack growth in nonlinear elasticity. Arch. Ration. Mech. Anal. 176 (2005), 165-225.

[11] A. Fiaschi. A Young measure approach to quasi-static evolution for a class of material models with nonconvex elastic energies. ESAIM Control Optim. Calc. Var. 15 (2009), 245-278.

[12] A. Fiaschi. Rate-independent phase transitions in elastic materials: a Young-measure approach. Netw. Heterog. Media 5 (2010), 257-298.

[13] I. Fonseca, D. Kinderlehrer, P. Pedregal. Energy functionals depending on elastic strain and chemical composition. Calc. Var. PDEs 2 (1994), 283-313.

[14] G. Francfort, A. Garroni. A variational view of partial brittle damage evolution. Arch. Rational Mech. Anal. 182 (2006), 125-152.

[15] M. Frémond. Non-Smooth Thermomechanics. Springer-Verlag, Berlin, 2002.

[16] A. Garroni, C. Larsen. Threshold-based quasi-static brittle damage evolution. Arch. Rational Mech. Anal. 194 (2009), 585-609.

[17] M. Giaquinta, E. Giusti. Quasi-minima. Ann. Inst. H. Poincaré (Analyse non lineaire) 1 (1984), 79-107.

[18] E. Giusti, Direct methods in the calculus of variations. World Scientific Publishing Co., Inc., River Edge, NJ, 2003.

[19] D. Kinderlehrer, P. Pedregal. Gradient Young measures generated by sequences in Sobolev spaces. J. Geom. Anal. 4 (1994), 59-90.

[20] M. Kružík, A. Mielke, T. Roubíček, Modelling of microstructure and its evolution in shape-memory-alloy single-crystals, in particular in CuAlNi. Meccanica 40 (2005), 389-418.

[21] A. Menzel, P. Steinmann. A theoretical and computational framework for anisotropic continuum damage mechanics at large strains. Int. J. Solids Struct. 38 (2001), 9505-9523.

[22] C. Miehe. Discontinuous and continuous damage evolution in Ogden-type large-strain elastic materials. Eur. J. Mech. 14 (1995), 697-720.

[23] C. Miehe, J. Keck. Superimposed finite elastic-viscoelastic-plastoelastic stress response with damage in filled rubbery polymers. Experiments, modelling and algorithmic implementation. J. Mech. Phys. Solids 48 (2000), 323-365. 
[24] A. Mielke, Flow properties for Young-measure solutions of semilinear hyperbolic problems. Proc. R. Soc. Edinb. Sect. A 129 (1999), 85-123.

[25] A. Mielke, Evolution of rate-independent inelasticity with microstructure using relaxation and Young measures. IUTAM Symposium on Computational Mechanics of Solid Materials at large Strains (Stuttgart, 2001), Solid Mech. Appl., 108 (2003), 33-44.

[26] A. Mielke, Deriving new evolution equations for microstructures via relaxation of incremental minimum problems. Comput. Methods Appl. Mech. Engrg. 193 (2004), 5095-5127.

[27] A. Mielke, T. Roubíček, A rate-independent model for inelastic behavior of shape-memory alloys. Multiscale Model. Simul. 1 (2003), 571-597.

[28] A. Mielke and T. Roubíček, Rate-independent damage processes in nonlinear elasticity. Math. Models Methods Appl. Sci. 16 (2006), 177-209.

[29] A. Mielke, T. Roubíček, U. Stefanelli. Г-limits and relaxations for rate-independent evolutionary problems. Calc. Var. PDEs 31 (2008), 387-416.

[30] A. Mielke, T. Roubíček, J. Zeman. Complete damage in elastic and viscoelastic media and its energetics. Comput. Methods Appl. Mech. Engrg. 199 (2010), 124-1253.

[31] A. Mielke, F. Theil. On rate-independent hysteresis models. NoDEA Nonlinear Differential Equations Appl. 11 (2004), 151-189.

[32] P. Pedregal, Parametrized measures and variational principles. Progress in Nonlinear Differential Equations and their Applications 30. Birkhäuser Verlag, Basel, 1997.

[33] M.O. Rieger, j. Zimmer. Young measure flow as a damage model. Z. Angew. Math. Phys. 60 (2009), 1-32.

[34] T.W. Ting. Generalized Korn's inequalities. Tensor 25 (1972), 295-302.

[35] M. Thomas, A. Mielke. Damage of nonlinearly elastic materials at small strain - Existence and regularity results. ZAMM Z. Angew. Math. Mech. 90 (2010), 88-112.

[36] M. Valadier, Young measures. Methods of nonconvex analysis (Varenna, 1989), 152-188, Lecture Notes in Math., Springer-Verlag, Berlin, 1990.

(Alice Fiaschi and Ulisse Stefanelli) IMATI-CNR, V. Ferrata 1, I-27100, Pavia, Italy

E-mail address, Alice Fiaschi: alice.fiaschi@imati.cnr.it

E-mail address, Ulisse Stefanelli: ulisse.stefanelli@imati.cnr.it

(Dorothee Knees) WIAS, Mohrenstrasse 39, D-10117, Berlin, Germany

E-mail address, Dorothee Knees: knees@wias-berlin.de 